\title{
Gradhiva
}

GRADHIV

Revue d'anthropologie et d'histoire des arts

$7 \mid 2008$

Le possédé spectaculaire

\section{Trois destinées, un destin}

Biographie d'une coiffure kwakwaka'wakw

Three destinies, one fate. Biography of a Kwakwa ka'wakw headdress

\section{Marie Mauzé}

\section{(2) OpenEdition}

Journals

Édition électronique

URL : http://journals.openedition.org/gradhiva/1084

DOI : $10.4000 /$ gradhiva. 1084

ISSN : 1760-849X

Éditeur

Musée du quai Branly Jacques Chirac

Édition imprimée

Date de publication : 15 mai 2008

Pagination : 100-119

ISBN : 978-2-915133-86-8

ISSN : 0764-8928

Référence électronique

Marie Mauzé, "Trois destinées, un destin », Gradhiva [En ligne], 7 | 2008, mis en ligne le 15 mai 2011, consulté le 19 avril 2019. URL : http://journals.openedition.org/gradhiva/1084 ; DOI : 10.4000/ gradhiva.1084

(c) musée du quai Branly 


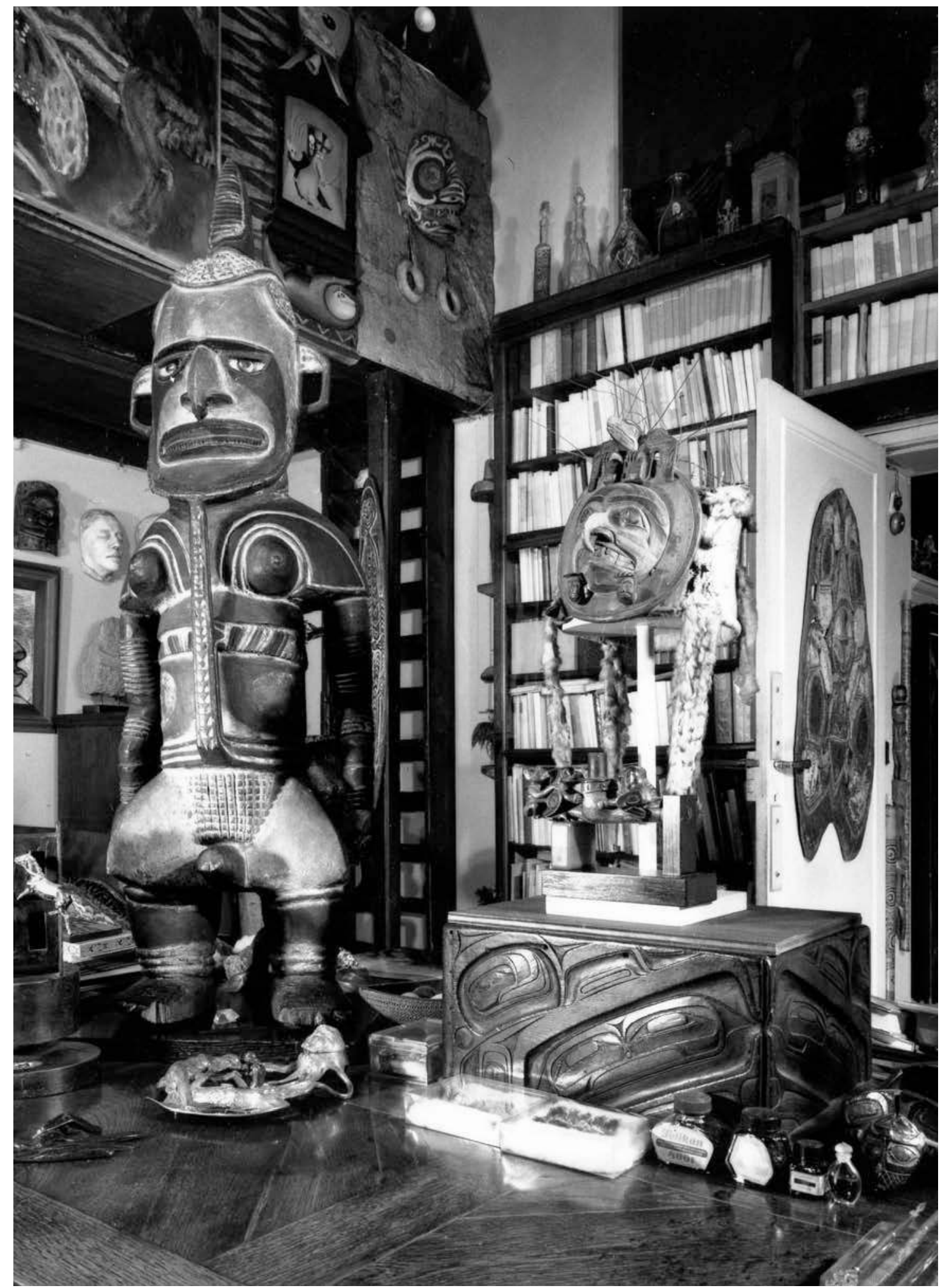

Fig. 1 Bureau d'André Breton avec la sculpture Uli de Nouvelle-Irlande $(\mathrm{H}: 125 \mathrm{~cm})$ et la coiffe kwakwaka'wakw $(\mathrm{H}: 18 \mathrm{~cm})$, sans date,détail. (C) Top-Rapho, photo Gilles Ehrmann. 


\title{
Trois destinées, un destin
}

\author{
Biographie d'une coiffure kwakwaka'wakw
}

\author{
Marie Mauzé
}

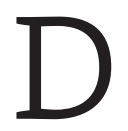
epuis une vingtaine d'années, en anthropologie comme en muséologie, un intérêt nouveau se manifeste pour l'étude de la culture matérielle (material culture), ce qui conduit ces deux disciplines à envisager la description-interprétation ou la présentation-représentation des objets sous un éclairage différent de celui qui prévalait jusqu'alors. À la suite notamment des travaux d'Arjun Appadurai (1986), il est couramment admis que les objets sont dotés d'une "vie sociale » inséparable de la manière dont les individus et les groupes les considèrent, aussi bien sur le plan matériel que symbolique ou imaginaire. Cette vie sociale est révélée par la multiplicité des regards portés sur ces objets : ils sont fabriqués par des artisans spécialisés ou des artistes pour un destinataire particulier ou collectif; immobiles ou en mouvement, ils occupent une place dans l'espace et dans le temps. Les objets naissent et disparaissent ou " meurent »; ils sont abandonnés, détruits, restaurés, et ont des fonctions et des significations différentes selon les contextes dans lesquels ils sont montrés, manipulés ou exposés.

Ainsi, l'objet ne doit pas être saisi seulement dans sa détermination singulière, mais selon une multiplicité de points de vue qui, dans la durée, lui donne ce qu'on appelle une " patine sociale " ou une " épaisseur sociale » (Inglis 1990, cité in Ames 1992 : 141; Ames ibid.; voir aussi Bonnot 2004:160) : les identités diverses attribuées à un objet au cours de sa vie ne s'éliminent pas les unes après les autres, au contraire elles se superposent en couches successives tant que l'objet existe sous sa forme matérielle. Pour indiquer qu'aucune « inscription » additionnelle n'efface ou ne voile une partie de son histoire, l'anthropologue Michael Ames (ibid.) utilise à ce propos la métaphore de l'objet palimpseste ; cette métaphore rend compte de la complexité des réseaux de relations qui se développent autour d'un objet en fonction du type de regard qui se pose sur lui : une même chose sera successivement objet sacré, objet d'échange ou marchandise, spécimen ethnographique, œuvre d'art, objet patrimonial. La liste n'est pas close des valeurs possibles, qui peuvent apparaître opposées, voire contradictoires (Appadurai 1986 : 56 ; Ames 1992 : 144). Comme le remarque Jean Jamin (2004 : 9) : «Un objet n'est pas la simple expression ou concrétion d'une relation; son existence matérielle pas plus que sa fonction ne préjugent de sa destinée culturelle. » Et Jamin d'ajouter : «En somme l'objet ne se réduit pas à la chose, celle-ci n'étant jamais que la totalité virtuelle de ses "objectivations" concurrentes ou successives. " 
Le propos de cet article est de présenter à la lumière du cadre conceptuel qui vient d'être défini la biographie d'un objet particulier, celle d'une coiffure cérémonielle kwakwaka'wakw (côte nord-ouest, Amérique du Nord), ce qui permettra de saisir à travers son parcours géographique et symbolique les différentes étapes de sa métamorphose (voir Kopytoff 1986 : 66-67).

\section{Historique : le Louvre ou le musée tribal?}

Le Louvre ou le musée tribal? La question peut paraître étrange quand on réfléchit sur le destin d'un objet tant ces deux options semblent a priori ne pouvoir jamais être mises en concurrence l'une avec l'autre. De l'atelier d'André Breton, où elle est restée quasiment ignorée pendant une quarantaine d'années, la coiffure cérémonielle kwakwaka'wakw (côte nord-ouest de l'Amérique du Nord) dont je retrace ici l'histoire aurait dû « aller » au Louvre (pavillon des Sessions) et rejoindre la centaine "de chefs-d'œuvre incontestables ${ }^{1}$ " choisis par Jacques Kerchache, alors commissaire de l'exposition permanente consacrée aux « arts premiers » inaugurée en avril 2000 dans le plus prestigieux des musées français. La coiffure est retournée, sinon dans sa communauté, du moins dans son pays d'origine en septembre 2003 grâce à la volonté d'Aube ElléouëtBreton et de sa fille Oona qui, ayant pris connaissance de son destin exceptionnel, ont décidé de la restituer aux Kwakwaka'wakw. Elle aurait pu devenir une œuvre valorisée pour ses qualités formelles et/ou sa force d'expression, elle a pris le statut d'objet patrimonial symbolisant la vitalité d'une culture autochtone.

Mais pourquoi cette pièce? Le choix de Kerchache s'est porté sur cette coiffure kwakwaka'wakw car, à coup sûr, il en appréciait la patine du bois d'aulne². Mais le plus important pour convaincre l'assistance de son bon jugement résidait dans le fait que la pièce avait un solide pedigree associé à une période précise de l'histoire de la sensibilité artistique des surréalistes vis-àvis des arts dit « sauvages ». En effet, la coiffure faisait partie de la collection André Breton, ce qui en justifiait la présence aux côtés des huit autres objets d'Alaska et de la côte nord-ouest jugés dignes de représenter l'Amérique du Nord au pavillon des Sessions : six pièces provenaient des anciennes collections de Max Ernst, de Claude Lévi-Strauss et de Breton (Mauzé 2000; Rousselot 2000). Ces œuvres ont une histoire singulière : elles ont été achetées par les amateurs passionnés d'art amérindien qu'étaient les surréalistes, pendant l'exil des années 1940 à New York, auprès du Museum of the American
Indian (Heye Foundation) par l'intermédiaire de l'antiquaire Julius Carlebach (Carpenter 1975, 2005 ; LéviStrauss 1983, 1988; Mauzé, 2004b, 2006).

Les notes laissées par Kerchache laissent penser que la coiffure avait suivi la même voie. Celui-ci tenait l'objet pour avoir fait partie de la collection de la sculptrice Isabelle Waldberg, membre du cercle surréaliste newyorkais, puis de celle du galeriste Claude Bernard avant d'en avoir fait lui-même l'acquisition lors de l'exposition Art primitif. Amérique du Nord qu'il organise en mai-juin 1965 pour la vendre à André Breton. Bien que cette filière ne soit pas confirmée aujourd'hui ${ }^{3}$, la coiffure faisait bien partie des collections du Museum of the American Indian avant d'avoir été acquise par le marchand d'art moderne Edward Primus, également expert en art précolombien à Los Angeles ; elle fut retirée de l'inventaire du musée en octobre $1957^{4}$. On ne sait donc pas précisément dans quelles circonstances la pièce est arrivée en France. En tout cas, l'objet est montré une première fois par Kerchache dans sa toute nouvelle galerie de la rue de Seine à l'occasion de l'exposition Arts primitifs (sans date), vraisemblablement en 1964. Le livret qui accompagne la manifestation comprend une reproduction en couleurs de la coiffure, identifiée comme

1. Expression utilisée par Jacques Kerchache dans un entretien avec Emmanuel de Roux, «Une centaine de chefs-d'œuvre "primitifs" vont entrer au Louvre », Le Monde, 15 juillet 1998.

2. Pour la petite histoire, il en a caressé le bois les doigts humectés de salive pour faire mieux apparaître la luminosité de la matière lors de la présentation de la pièce au petit comité ( Jacques Friedmann, Germain Viatte, Christiane Naffah et moi-même) réuni autour de lui au Louvre, un matin de début décembre 1998.

3. Sur la fiche établie en décembre 1998 par Jacques Kerchache dans le cadre d'une proposition d'achat soumise au comité de présélection des acquisitions (mission de préfiguration du musée de l'Homme, des Arts et des Civilisations, 5, rue Auguste Vacquerie, $\mathrm{XVl}$ e arrondissement, le 11 décembre 1998, proposition $n^{\circ} 1$ ], sont indiquées les informations suivantes: «Provenance : The Museum of the American Indian, Heye Foundation, New York ( $n^{\circ} 14.6883$ ), acheté par Carlebach. Anciennes collections Isabelle Waldberg, Claude Bernard et Jacques Kerchache. Collection André Breton. » Les éléments du pedigree établi par Kerchache faisant référence aux collections d'Isabelle Waldberg et de Claude Bernard ne sont confirmés ni par Michel Waldberg, fils d'Isabelle, ni par Claude Bernard (entretiens de l'auteur avec Michel Waldberg et Claude Bernard le 20 avril 2004 ; entretien avec Michel Waldberg le 23 janvier 2007). Dans le petit catalogue de l'exposition Art primitif. Amérique du Nord (1965), aucune référence n'est faite à la collection d'Isabelle Waldberg, alors que quelques pièces sont explicitement référencées « ancienne collection Isabelle Waldberg 》.

4. Ces informations sont indiquées sur la fiche d'inventaire du National Museum of the American Indian (Museum of the American Indian, Heye Foundation). Mes remerciements vont à Ann Drumheller (correspondance des 29 et 30 décembre 1998) et Maria MacWilliams (correspondance du 19 janvier 2007). Je remercie Ted Carpenter pour les informations qu'il m'a communiquées sur l'identité et la raison sociale d'Edward Primus (lettre à l'auteur datée du 28 novembre 2003). 


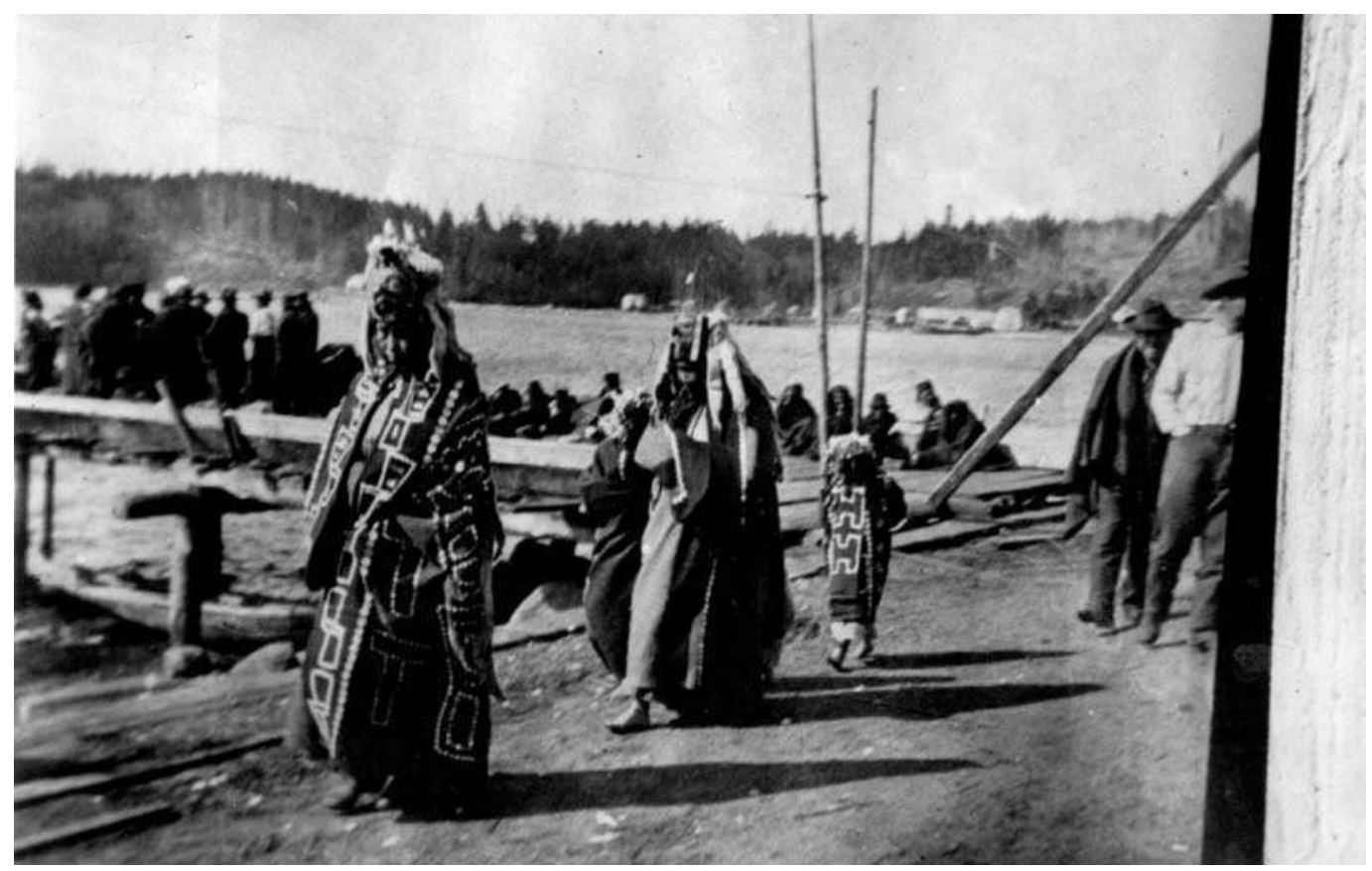

Fig. 2 Alert Bay vers 1910, deux chefs et un enfant en costume cérémoniel. () Image AA-00210, courtesy of Royal BC Museum, BC Archives.

« masque haida ${ }^{5}$ ». La pièce est à nouveau présentée en mai-juin 1965 dans l'exposition Art primitif. Amérique $d u$ Nord, qui réunit un ensemble de cinquante-trois pièces (Alaska, côte nord-ouest, Sud-Ouest ${ }^{6}$ ). On peut penser que Breton en fait l'acquisition le 19 juin 1965; c'est en tout cas, comme l'indique son agenda, la date à laquelle il prend rendez-vous avec Jacques Kerchache avant de quitter Paris pour sa maison de Saint-CirqLapopie. Un court article paru dans Le Monde du 5 juin 1965 sous la plume de Paule-Marie Grand, intitulé «Primitifs d'Amérique du Nord », a retenu son attention, et tout particulièrement un paragraphe décrivant la coiffure, que Breton a encadré à l'encre rouge?.

Cependant, la coiffure avait gardé une part de mystère que Kerchache ne pouvait pas soupçonner. Un examen attentif a permis de préciser l'origine ethnique de l'objet, tandis que le numéro d'inventaire inscrit sur sa face interne a signalé son appartenance aux collections du Museum of the American Indian fondé par le collectionneur George Heye en 1916 et la date de son entrée dans ce musée. Cette information et un ensemble de photographies d'archives ont révélé la véritable identité de la pièce, ce qui empêchait son acquisition par l'État français car, étant donné son histoire, elle ne pouvait manquer d'être soumise à une demande de restitution de la part des Kwakwaka'wakw (Mauzé 2004a : 116). La coiffure a eu une trajectoire singulière qu'il a été possible de saisir sur presque un siècle. Elle fait partie d'une collection d'objets confisqués aux Kwakwaka'wakw par le gouvernement canadien en 1922 à la suite de l'organisation illégale d'un potlatch, une loi de 1884 interdisant toute cérémonie ayant à voir avec la distribution de biens ou toute initiation dans un rituel accompagnée de chants, de danses et de discours ${ }^{8}$.

La « Potlatch Collection » ou " Alert Bay Collection », d'après le nom de la communauté où elle a été rassemblée, réunit quelque cinq cents objets appartenant à des chefs et des nobles issus de neuf tribus kwakwaka'wakw ${ }^{9}$. Les objets confisqués ont été par la suite

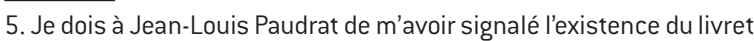
intitulé Arts primitifs. Le cliché en couleurs publié dans le petit catalogue est utilisé pour illustrer l'objet dans la notice rédigée pour le comité de présélection (information de Jean-Louis Paudrat).

6. La date de l'exposition est indiquée non pas dans le catalogue mais sur l'affiche de l'exposition (voir Bethenod 2003 : 27). Parmi les pièces exposées, quelques-unes appartiennent au prince Saddrudin Aga Khan, d'autres à André Breton et Robert Lebel, quelques-unes proviennent de l'ancienne collection d'Isabelle Waldberg.

7. Une photocopie de l'article et une photocopie d'une page de l'agenda de Breton m'ont été communiquées par Jean-Michel Goutier le 10 mai 2004. Je l'en remercie très chaleureusement.

8. Dans le cadre de cet article, il n'est pas possible d'expliquer en détail les fondements de la loi anti-potlatch, ni les modalités de son application, ni les circonstances précises de la confiscation. Voir Bracken 1997; Cole et Chaikin, 1990 ; Mauzé 1992, 1995, 1999a ; Sewid-Smith 1979.

9. Il est impossible de connaître le nombre exact de pièces confisquées : les chiffres oscillent entre quatre cents et sept cents. Le National Museum d'Ottawa l'a estimé à cinq cent soixante-seize objets (voir Saunders 1997 : 119-120, note 16). 


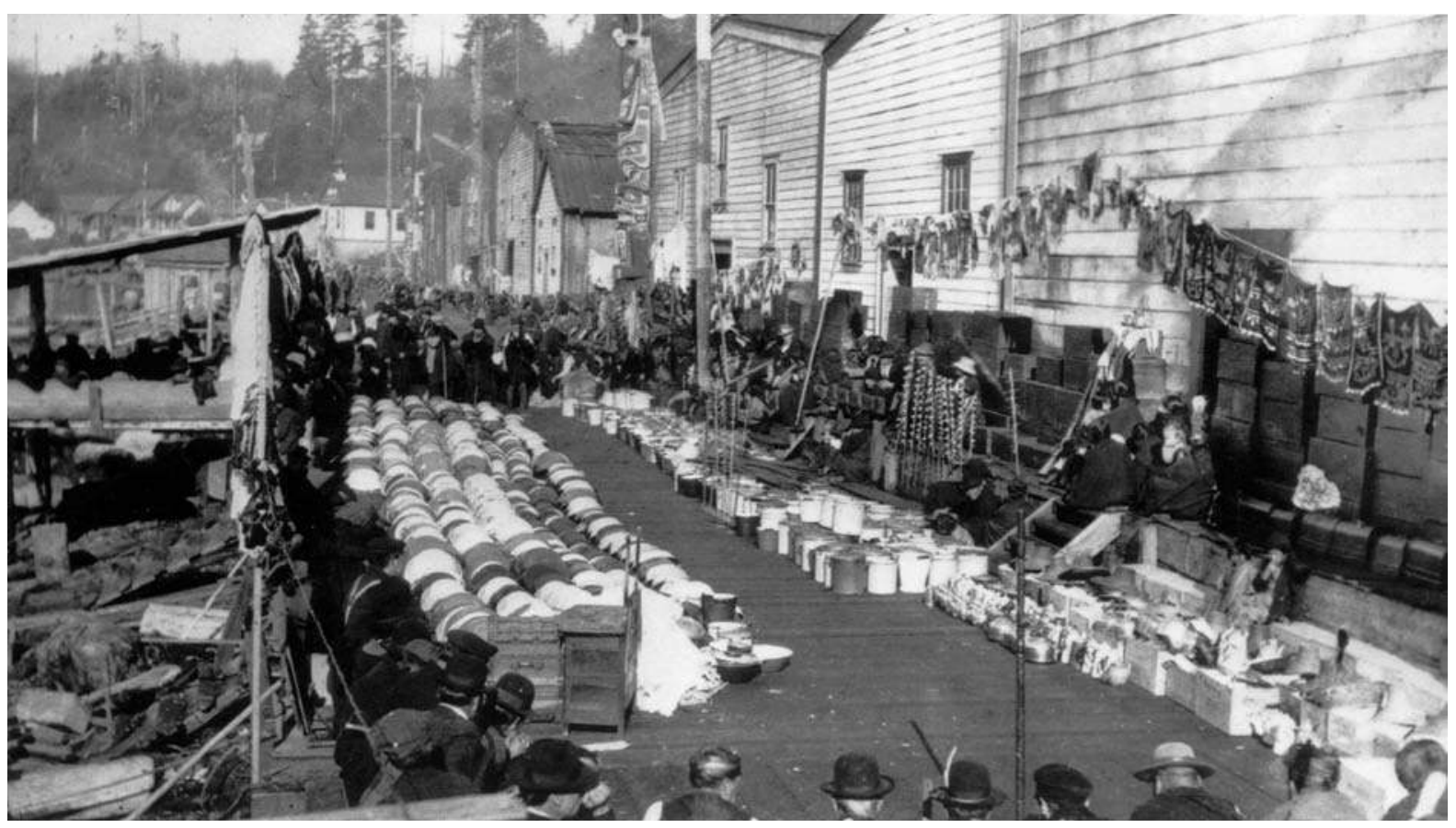

Fig. 3 Carte postale d'un potlatch du chef et sculpteur Bob Harris à Yalis (Alert bay) vers 1910. Sont exposés les biens qui vont être distribués dont des bassines et des seaux en émail, des coiffures, des tabliers cérémoniels et des bijoux empilés sur des bâtons. @ Image AA-00009, courtesy of Royal BC Museum, BC Archives.

répartis principalement entre deux musées d'ethnographie canadiens : le National Museum (à l'époque Victoria Memorial Museum) d'Ottawa, aujourd'hui musée canadien des Civilisations, et le Royal Ontario Museum à Toronto. Ces acquisitions représentaient l'occasion pour ces deux institutions d'enrichir leurs collections, plutôt pauvres à l'aune de celles des musées d'ethnographie des États-Unis et d'Europe (d'Allemagne notamment), les collectes massives d'artefacts en provenance de la côte nord-ouest ayant été entreprises dans le dernier quart du xixe siècle à leur profit (Cole 1985). Quelques pièces vont connaître un sort différent de celui prévu par l'administration. En septembre 1922, l'agent des Affaires indiennes, William Halliday, contrairement à son propre engagement et aux directives du département des Affaires indiennes, vend à bon prix à George Heye trente-cinq pièces exceptionnelles. Ce dernier sera en mesure d'acquérir en 1926 quelques pièces supplémentaires appartenant à la Potlatch Collection détournées par Donald Angermann, le policier qui avait procédé à l'arrestation des chefs kwakwaka'wakw. La coiffure cérémonielle fera partie du nombre ${ }^{10}$.

Quelque cinquante années après la confiscation, des représentants des Kwakwaka'wakw demandèrent le retour des pièces que le gouvernement canadien s'était abusivement appropriées. Après de longues négociations entre les Kwakwaka'wakw, le département des
Affaires indiennes et le National Museum, il a été décidé en 1973 par les instances canadiennes - en dépit du mécontentement des autochtones - qu'en vertu de la restitution les objets prenaient désormais statut d'éléments du patrimoine culturel et en conséquence devaient être accueillis dans un musée local chargé de les conserver selon les règles en vigueur au Canada. À la suite de dissensions internes aux communautés autochtones concernées, la collection restituée par le National Museum a été répartie entre le Kwagiulth Museum and Cultural Centre de Cape Mudge, inauguré en juin 1979, et le U'Mista Cultural Centre d'Alert Bay, ouvert en novembre 1980. En 1988, quelque cent trente pièces restituées par le Royal Ontario Museum sont réparties entre les deux musées. Dans le cadre de sa politique de rapatriement, le National Museum of the American Indian, par la voix de son directeur Richard West, s'était engagé à retourner les pièces de la Potlatch Collection en sa possession. Une partie des objets acquis par Heye en 1922 et 1926 a été restituée aux Kwakwaka'wakw en 1993 et 2002, au bénéfice du U'Mista Cultural

10. Au moins trois objets ont été « prélevés » de la Potlatch Collection par Donald Angermann dans des circonstances qui nous sont inconnues. Ces pièces ne figurent sur aucun inventaire, mais ont été photographiées par William Halliday. Un ensemble de treize objets a été vendu par l'épouse d'Angermann à Heye en 1926. Elles ont été inventoriées par ce dernier sous le nom de «B.E. Angermann Collection 》 (U'Mista Cultural Centre Archives, File U 96-032-30-13). 
Centre ${ }^{11}$. En novembre 2006, à l'occasion du vingt-cinquième anniversaire de cet établissement, le British Museum a accordé à ce dernier un prêt à long terme pour un masque de la Potlatch Collection acheté par Heye en 1922 et entré dans ses collections en 1944. C'est aussi au U'Mista Cultural Center qu'Aube Elléouët-Breton et sa fille Oona, après avoir pris connaissance des circonstances dramatiques de la confiscation de la Potlatch Collection, ont rendu aux Kwakwaka'wakw en septembre 2003 la coiffure que Kerchache convoitait pour l'exposition du Louvre (Mauzé 2004a : 216-217).

Revenons en arrière : la coiffure a été conservée au Museum of the American Indian pendant plus de trente ans, de 1926 à 1957. Cette période a été celle du « grand sommeil », selon l'expression bienvenue de Feest (1998: 257). Comme plusieurs milliers de spécimens, elle est restée dans l'anonymat des réserves de l'annexe du Bronx sans jamais avoir été exposée ni prêtée à l'occasion d'expositions temporaires ${ }^{12}$. La coiffure n'a d'existence que par la fiche d'inventaire qui fait office de carte d'identité. La véritable passion de Heye était d'accumuler le plus grand nombre de pièces de manière à enrichir constamment sa collection -à sa mort en janvier 1957, elle est évaluée à environ un million d'objets des deux Amériques. Tout autant que des spécimens ethnographiques, les objets étaient considérés comme des marchandises destinées à être échangées contre d'autres qui remportaient la faveur du moment. En vérité, Heye ne manifestait aucun intérêt particulier pour les sociétés dont provenaient les pièces. Il n'avait pas non plus l'ambition de préserver un patrimoine menacé de disparition -il lui arrivait même de se séparer de la documentation relative à des collections privées qu'il acquérait en totalité. L'éducation du public était le cadet de ses soucis - songeons que le musée n'était ouvert que trois heures par jour et que les salles d'exposition étaient surtout remarquables pour l'entassement de pièces trop nombreuses dans des vitrines de style victorien sombres et poussiéreuses ${ }^{13}$ (Carpenter 2005 : 25-26, 48; Wallace 1960 : 104-105, 107).

Sortie de cet endroit pour être admirée par Breton, la coiffure sera vue et appréciée par de nombreux visiteurs, accueillis dans l'atelier par sa femme Élisa après la mort de celui-ci. Est-elle arrivée au bout de la route qui l'a reconduite chez elle? Voilà brièvement reconstituée l'histoire de la coiffure entre le moment de sa confiscation, en 1922, et celui de sa restitution, en 2003, après des stations plus ou moins longues : Alert Bay (Colombie-Britannique), New York, Los Angeles, Paris, Alert Bay. Les principales étapes de ce parcours consti- tuent autant de balises spatio-temporelles qui définissent les transformations de statut et de signification que l'objet a éprouvées au cours de son histoire. L'analyse de l'histoire de vie de cette pièce fait apparaître des configurations de relations mises en place entre le pouvoir colonial et les autochtones à la fin du XIX et au début du xx siècle, la politique de collecte des musées d'ethnographie, l'appropriation par les collectionneurs d'objets promus au rang d'œuvres d'art et le rapport qu'entretiennent aujourd'hui les Premières nations canadiennes avec leur patrimoine culturel.

\section{Dlugwe' - ou bien précieux}

De la vie antérieure de la coiffure, on sait peu de choses, si ce n'est que ses caractéristiques formelles et ce qu'elle donne à voir informent sur son usage rituel dans le contexte de manifestations cérémonielles dites dluxwala ou tla'sala, au cours desquelles sont présentés des masques illustrant des événements relatifs à la rencontre d'un ancêtre avec des êtres surnaturels dispensateurs de richesses et de privilèges dans les temps mythiques. Avant l'entrée des masques, a lieu la danse de la coiffure (headdress dance), où un jeune initié apparaît dans la maison cérémonielle vêtu d'une couverture blasonnée et portant une coiffure dite yaxwi'we en kwak'wala $^{14}$. La coiffure dont il est question ici est composée d'une plaque frontale de forme arrondie figurant un faucon aux traits anthropomorphes surmonté d'un corbeau aux ailes déployées. La tête du faucon est flanquée de deux serres prenant appui sur un visage de petite taille sculpté en relief, les dents et les yeux rehaussés de fragments d'haliotide dont l'éclat est conçu comme la manifestation tangible du pouvoir surnaturel dont sont investis les chefs (Mauzé 1999b). Le pourtour de la

11. Neuf pièces ont été restituées en 1993 par le National Museum of the American Indian (dont deux inventoriées sous le nom de B.E. Angermann) et dix-sept en 2002 (voir U'Mista News, hiver 2002 ; U'mista Cultural Centre Archives, Files U96-032-30-12 a, U96-03J-30-10, U00032-30-12).

12. C'est en 1926 que Heye fait construire une annexe au musée, trop petit pour contenir sa riche collection alors constituée d'environ quatre cent mille pièces (Force 1999: 9). Aucun document ne fait état de prêt ou d'exposition pour cet objet.

13. Ce n'est qu'en 1956, avec l'arrivée au Museum of the American Indian de l'ethnologue Frederick J. Dockstader, que la muséographie est renouvelée à l'aide de vitrines mieux éclairées et moins encombrées. Selon Wallace (1960: 104), Dockstader retire quatre objets sur cinq des vitrines, rédige de nouveaux cartels et s'attache à mettre en évidence les pièces importantes de la collection.

14. Yaxwi'we signifie «masque de danse porté sur le front ». Le rituel dluxwala appartenait à l'origine aux tribus septentrionales. 
coiffure est complété par une couronne de barbillons d'otarie et une longue traîne en peaux d'hermine. La coiffure appartient à la catégorie des dlugwe', « trésors ", comprenant des blasons, des accessoires rituels et des masques. Propriétés individuelles d'un chef ou d'un personnage de haut rang, les coiffures et les masques possèdent une identité singulière définie par leur nom et le mythe auquel ils sont indissociablement liés.

Il n'en demeure pas moins que l'objet, si l'on peut dire, est muet sur son origine. On sait seulement qu'il a été soustrait de la Potlatch Collection avant même qu'un inventaire sommaire en ait été établi, mais après que les pièces ont été photographiées ${ }^{15}$. L'histoire documentaire d'un objet ethnographique prend généralement forme au moment où l'objet entre dans les collections d'un musée (Feest 1998). L'établissement de la fiche d'inventaire permet de rassembler les informations nécessaires à son identification : provenance, date de collecte, nom du collecteur, date d'entrée dans le fonds du musée. Dans le cas présent, les documents photographiques constituent les premiers éléments de sa carte d'identité.

\section{Objets cérémoniels et politique indienne}

Avec la confiscation de la Potlatch Collection se joue une opération qui met en évidence les relations conflictuelles entre pouvoir colonial «étranger » et monde autochtone, la Potlatch Collection illustrant de manière explicite la nature de ces relations. Rappelons que les objets ont été confisqués sur la proposition de Donald Angermann, le policier qui avait joué un rôle clé dans l'arrestation des chefs et nobles kwakwaka'wakw et avait mené l'instruction des poursuites judicaires avec beaucoup de zèle. Si William Halliday était prêt à adopter une solution de compromis, Angermann souhaitait porter l'affaire jusqu'à son terme, car en dépit de leurs promesses, les Indiens ne s'étaient jamais résolus à cesser leurs activités délictueuses. " Pour prouver [leur] bonne foi ", Angermann suggère que les coupables « abandonnent de leur plein gré les cuivres, les masques, les coiffures et tout autre attirail utilisé uniquement dans les potlatch » et convainquent les membres de leur communauté non inculpés dans le potlatch de 1921 de faire de même en signe de solidarité (Cole et Chaikin 1990 : 120-121; Halliday 1935 : 191 ; Mauzé 1999a : 421). Le résultat de cet accord permet aux Kwakwaka'wakw d'acheter leur liberté en cédant au département des Affaires indiennes leurs biens cérémoniels. Il permet en outre au gouvernement cana- dien de trouver une porte de sortie acceptable dans le respect de la loi sans que de lourdes peines d'emprisonnement soient prononcées. Par son existence même, la Potlatch Collection témoigne des relations asymétriques de pouvoir qui se sont développées entre administration coloniale et autochtones. Cependant, se pose la question de la légitimité du détournement de plusieurs pièces - au moins cinq, dont la coiffure - par Angermann, probablement avec l'accord de Halliday ${ }^{16}$. Tout se passe comme si ces objets " mis de côté " avaient statut de récompense matérielle et symbolique pour services rendus par Angermann à l'État canadien. Dans ce contexte, si elle partage un destin commun avec les objets de la Potlatch Collection, la coiffure est marquée du sceau du détournement, lequel apporte une dimension supplémentaire à son histoire.

Au-delà de ces considérations politiques, on observe qu'à l'instar des musées, le gouvernement canadien avait faite sienne l'idée selon laquelle la culture est fondée en partie sur des objets matériels. Partant de cette hypothèse, la confiscation avait pour but de priver les Kwakwaka'wakw des supports matériels de leurs pratiques cérémonielles et religieuses au fondement de leur système social. Du point de vue autochtone, la disparition des objets rituels n'entraîne pas la perte des droits et des privilèges ancestraux dont ils se prévalent; les choses ne sont que représentation matérielle de biens immatériels, ceux-ci n'ayant d'existence que pour autant qu'ils sont associés à une histoire, qu'un mythe leur donne vie, qu'une pratique les « met en acte " à travers l'exécution de chants et de danses (Gloria Webster in Laforet 1993 : 28-29). Certes, la confiscation de la Potlatch Collection a été vécue comme un événement très traumatisant par les Kwakwaka'wakw, et la mémoire de ce qui justifiait leur présence au monde s'est délitée au fil des ans, moins en raison de l'absence d'objets que de l'interdiction des pratiques rituelles permettant d'en actualiser la présence.

15. La coiffure apparaît sur trois photographies (PN 12209, PN 111636, PN 9923. Victoria, Royal British Columbia Museum, Ethnology Division).

16. Un état a été établi par Mary Jane Lenz du National Museum of the American Indian (U'Mista Cultural Centre Archives UCC U96-032-30-13; voir aussi «Regalia Notes » by Randall Macnair, U'Mista Cultural Centre Archives, UCC U96-032-30-09]. On peut penser que William Halliday a lui-même informé George Heye de l'origine de quelques-unes des pièces se trouvant dans la collection de B.E. Angermann et qu'il a acquises lors de son voyage en Colombie-Britannique de 1926. Cette hypothèse a été formulée par Joseph $\mathrm{H}$. Schaeffer in Research of records to determine the origins of the B.E. Angermann Collection, "prepared for the Province of British Columbia, Ministry Attorney General, Police Services Branch", 16 octobre 1992 (UCC U96-032-30-13). 


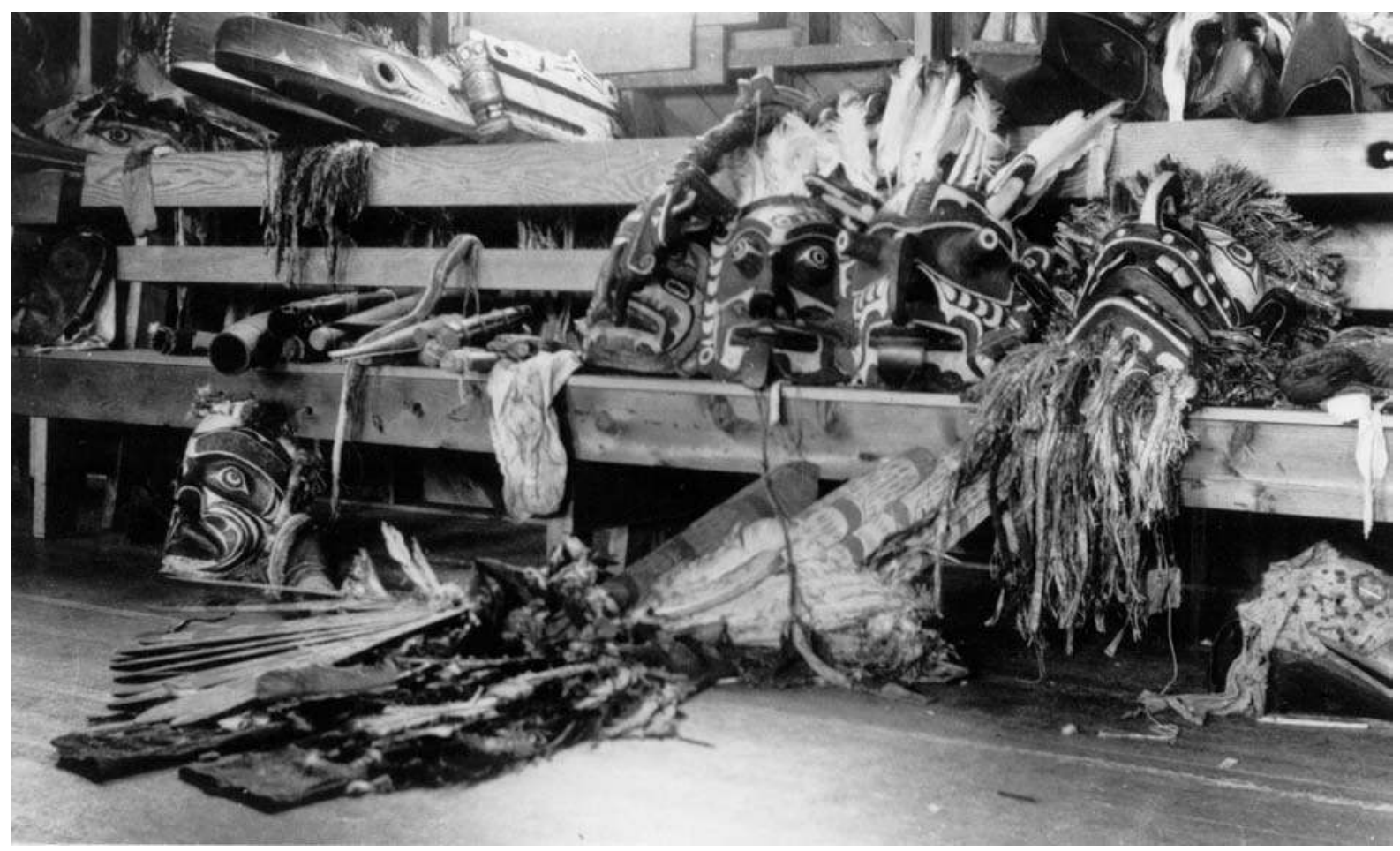

Fig. 4 Masques de la Potlatch Collection. Cliché Révérend V.S. Lord, 1922. @ Image PN 116 46, courtesy of Royal BC Museum, BC Archives.

\section{Confiscation et fabrication de l'objet ethnographique}

La confiscation est le "geste décisif » (Schaeffer 2004: 33) qui a opéré une coupure irréversible dans le destin de la coiffure et des quelque cinq cents pièces qui partagèrent pour quelques semaines un sort commun. D'objets cérémoniels faisant partie d'un patrimoine individuel ou familial, associés à un savoir mythique et ésotérique (Harkin 2005 : 19; Townsend-Gault 1997 : 142), ils prennent le statut, sous l'effet d'une collecte " forcée " entreprise à grande échelle à des fins d'éradication de la culture traditionnelle, de spécimens ethnographiques, fonction à laquelle ils n'étaient pas originellement destinés. Comme le souligne Barbara KirshenblattGimblett :

«Les artefacts ethnographiques sont des objets d'ethnographie [...]. Ils deviennent ethnographiques parce qu'ils ont été définis comme tels, détachés, coupés [de leur contexte d'origine] et emportés par les ethnographes. Ils sont ethnographiques non pas parce qu'on les a trouvés dans une maison paysanne hongroise, un village kwakiutl ou un marché du Rajasthan plutôt qu'au palais de Buckingham ou dans l'atelier de Michel-Ange, mais en raison de la manière dont ils ont été extraits de leur milieu d'origine [...] par les ethnographes $[\ldots]$ » $(1991: 387,1998: 17-18)$.
Des objets cérémoniels ou de vulgaires bouts de bois? Les pièces confisquées, dont le volume total est estimé à trois cents pieds cubes, ont été dans un premier temps entreposées dans la remise à bois de l'agent des Affaires indiennes. Elles ont été ensuite exposées dans la salle paroissiale attenante à l'église anglicane d'Alert Bay, sommairement inventoriées et photographiées avant d'être placées dans des malles destinées au musée d'Ottawa, où elles ont été inventoriées une nouvelle fois et évaluées en vue du versement par le département des Affaires indiennes d'une compensation aux chefs qui se sont défaits de leur regalia de potlatch ${ }^{17}$. En avril 1922, William Halliday présente la situation dans les termes suivants :

17. Les pièces ont été réunies tribu par tribu, souvent avec l'aide des missionnaires comme ce fut le cas dans la communauté lekwiltoq de Cape Mudge avec le révérend R.C. Scott. II semblerait qu'un premier inventaire ait été établi par l'instituteur d'Alert Bay au moment où les pièces ont été présentées dans la salle paroissiale (voir Saunders 1997 : 99). Nous disposons des inventaires établis sur les recommandations de Halliday par le personnel du Victoria Memorial Museum sous la direction d'Edward Sapir. L'ensemble de la collection a été estimé à mille quatre cent cinquante-six dollars canadiens. Les Kwakwaka'wakw ont été dédommagés à raison de cinq à dix dollars par masque, sauf les cuivres, qui étaient considérés sans «valeur ethnologique » par Halliday et ne pouvaient être remboursés à leur valeur réelle. Certains objets étaient estimés à dix mille dollars (Cole 1985 : 252-253; Hunt 1924 ; Mauzé 1999a : 421-422]. 


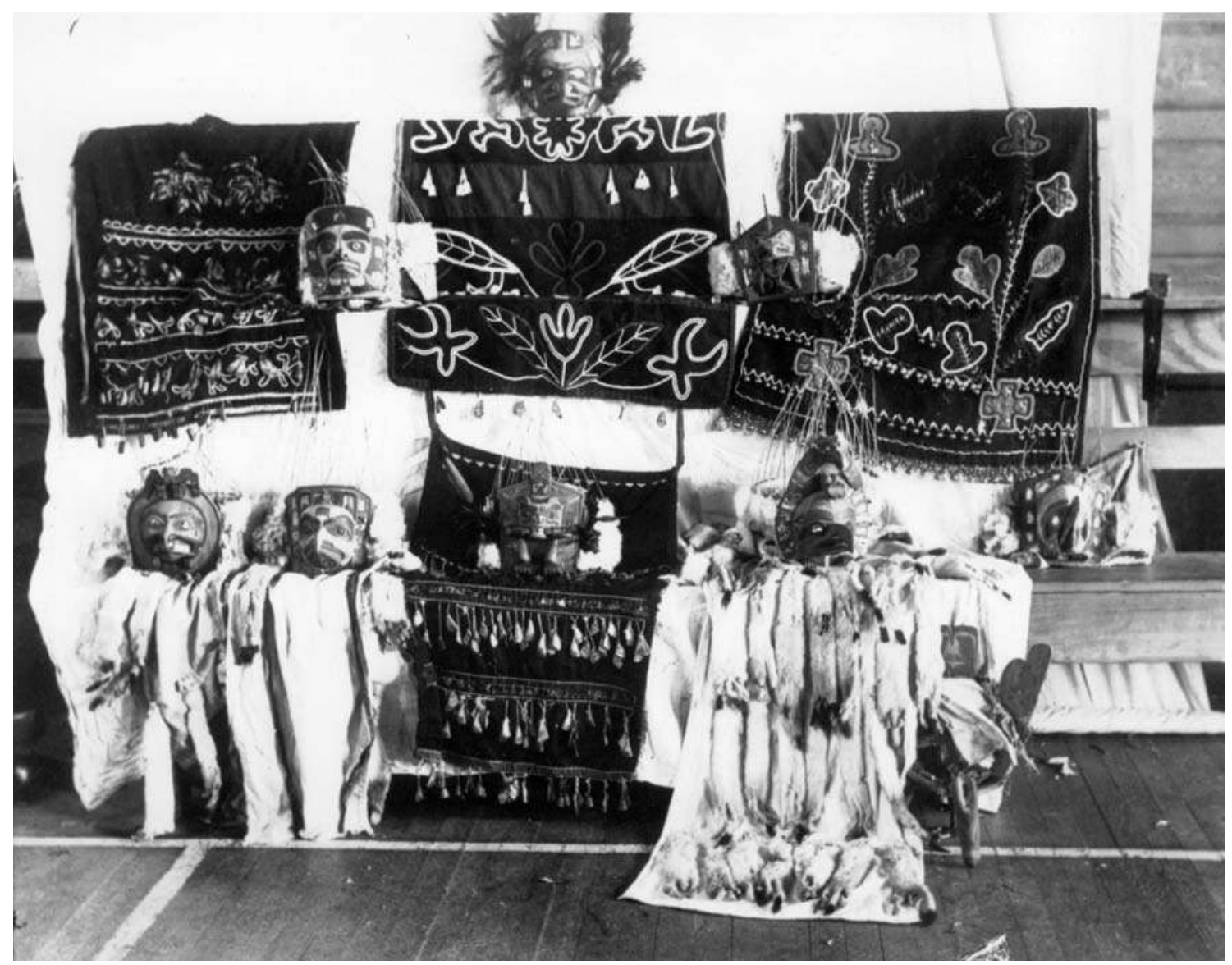

Fig. 5 Exposition des pièces de la Potlatch Collection dans la salle paroissiale d'Alert Bay. Sont réunies sur la photographie des objets appartenant à la cérémonie tla'sala. La coiffure "Breton » est en bas à gauche. Cliché William Halliday, 1922. @ Image PN 9923, courtesy of Royal BC Museum, BC Archives.

Je me suis arrangé avec l'Église anglicane pour obtenir l'usage de la salle paroissiale afin de déballer les objets et d'en établir la liste, la condition étant que nous puissions les exposer pendant quelques jours pour payer la location de la salle ${ }^{18}$.

\section{Et Halliday de poursuivre :}

Une fois l'exposition mise en place, j'ai l'intention de prendre quelques photographies des différents types de pièces car cette occasion ne se représentera certainement pas (cité in SewidSmith 1979 : 39).

Dissociés du potlatch, qui est considéré alors comme une institution scandaleuse chargée de tous les maux, les objets deviennent "inoffensifs " par l'artifice de la " décontextualisation "; ils ont vocation à représenter, selon les mots de Halliday, " [...] une collection de très grande valeur et très rare qui devrait se vendre cher dans le cadre des musées " (1935). À ce titre, l'agent recommande que les différents musées canadiens soient informés de l'existence de cette précieuse collection de sorte qu'elle reste au Canada et entre dans le patrimoine national (ibid.), recommandation qu'il n'hésitera pas à enfreindre en vendant trente-cinq pièces à George Heye, sous prétexte que celui-ci en offrait un bon prix ${ }^{19}$. L'attitude de Halliday peut paraître paradoxale, mais ce n'est pas le cas : l'abandon des objets cérémoniels est le prix à payer par les Kwakwaka'wakw pour éviter la prison, mais Halliday, soucieux des conditions économiques des Indiens dont il a la charge et conscient de la valeur marchande des objets, souhaite que les tribus obtiennent en compensation le plus d'argent possible.

L'exposition dans la salle paroissiale parachève le changement de statut des objets cérémoniels en objets ethnographiques. L'analyse des clichés pris à cette occasion par l'agent des Affaires indiennes et par le révé-

18. Le droit d'entrée est fixé à vingt-cinq cents, ce qui à l'époque représentait une somme relativement importante (Sewid-Smith 1979: 92).

19. Heye fait l'acquisition des trente-cinq pièces pour un montant total de deux cent quatre-vingt-onze dollars. 
rend Vivian Lord, en poste à Alert Bay, montre que la présentation des objets est plus artisanale que professionnelle. L'exposition a été montée avec les moyens du bord : un drap blanc délimite l'espace d'exposition et fournit l'arrière-plan "neutre » nécessaire à la prise des clichés; des bancs et le sol font office de vitrines ouvertes ${ }^{20}$. Les regalia sont regroupés non pas en fonction de leur appartenance familiale ou tribale, mais par série ou type, à la manière dont ils auraient pu être montrés dans les vitrines d'un musée. Il est remarquable que la typologie élaborée par l'agent des Affaires indiennes repose sur des catégories assez floues, inspirées par des critères de ressemblance formelle : ainsi sont présentés dans un même ensemble des masques appartenant à chacun des deux principaux complexes rituels (tseka et tla'sala), l'un relevant des initiations dans les sociétés secrètes, l'autre du pouvoir sacré des chefs et des relations avec les ancêtres. Sont posés côte à côte des masques anthropomorphes ou zoomorphes représentant des oiseaux et des mammifères marins, et plusieurs pièces de grande taille. Des ensembles plus cohérents apparaissent dans la mise en séries d'objets de même type : cuivres ou coiffures, par exemple. On observera que quelques pièces sont portées par un «figurant » à l'allure complètement figée, les photographies ressemblant étrangement aux clichés utilisés par les muséographes du début du xxe siècle pour mettre en scène les mannequins costumés des dioramas ${ }^{21}$. On a bien affaire là à une exposition unique en son genre, marquée par l'esprit des lieux; elle revêt un caractère exemplaire dans le souci de documenter de manière quasi exhaustive l'ensemble de la collection par le moyen de la photographie : le décor demeure, les objets tournent par séries successives. Bien que nous ayons affaire à une muséification temporaire à visée strictement documentaire, l'exposition se donne à voir comme l'acte crucial

20. Le même type de présentation avait été employé par Halliday en 1914 lors de la commission mise en place par le département des Affaires indiennes (1913-1916) en vue de l'établissement définitif des réserves en Colombie-Britannique (Savard 2005 : 73-74, 87).

21. Une photographie présente un chef nommé John Drabble vêtu d'une couverture blasonnée, coiffé d'un cimier et exhibant deux cuivres, un masque posé de chaque côté de ses pieds; un autre cliché présente un danseur arborant un costume hamatsa (voir aussi British Columbia Archives H-03995).

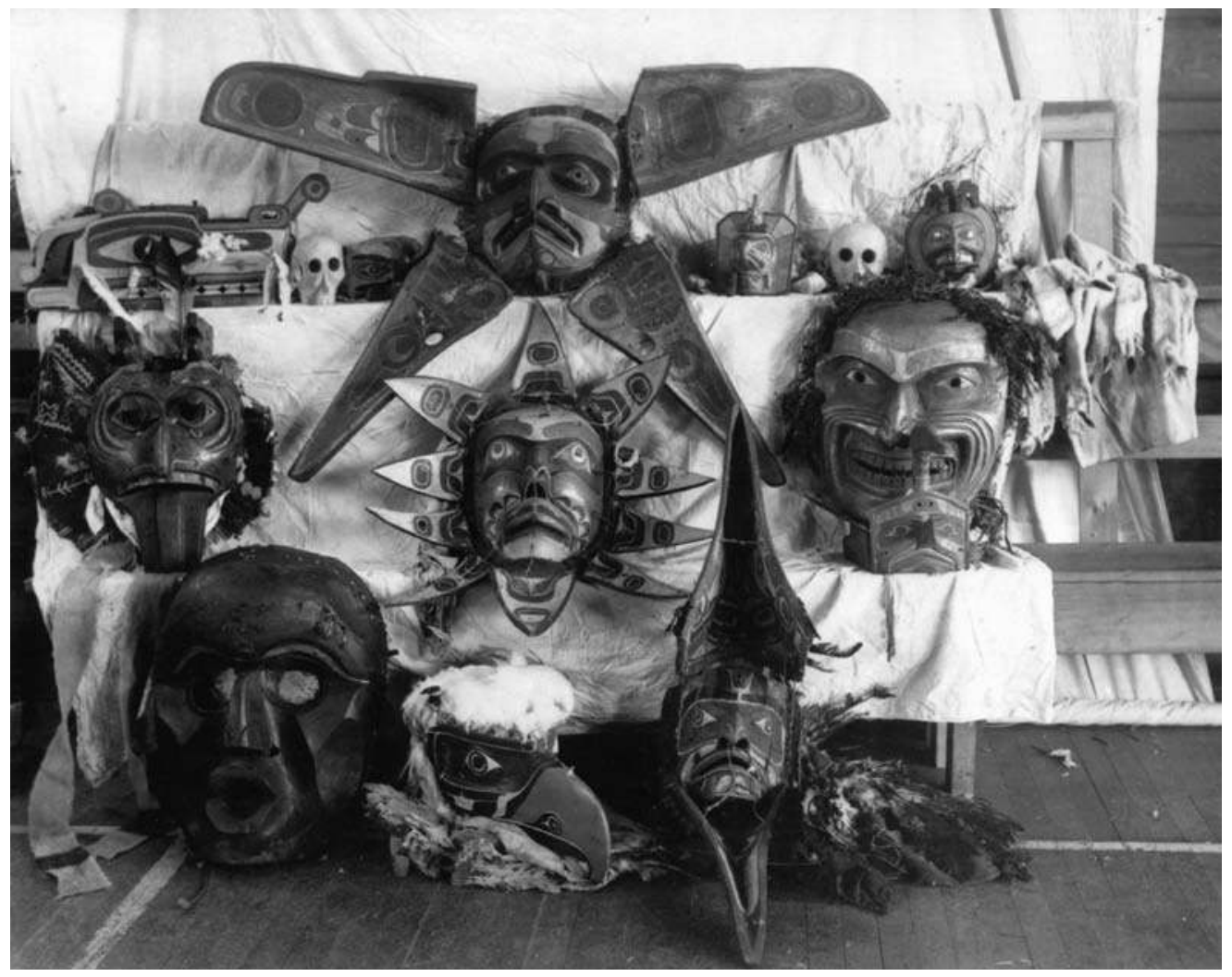

Fig. 6 Masques de la Potlatch Collection exposés dans la salle paroissiale d’Alert Bay. La coiffure "Breton " se trouve en haut à gauche. Au centre deux masques à transformation. Le masque comportant quatre volets (en haut) a été acheté par Heye en 1922. Il a été acquis par le critique d'art Georges Duthuit en 1944 lors de son exil new-yorkais. Cliché William Halliday, 1922. @ Image AA-00211, courtesy of Royal BC Museum, BC Archives. 


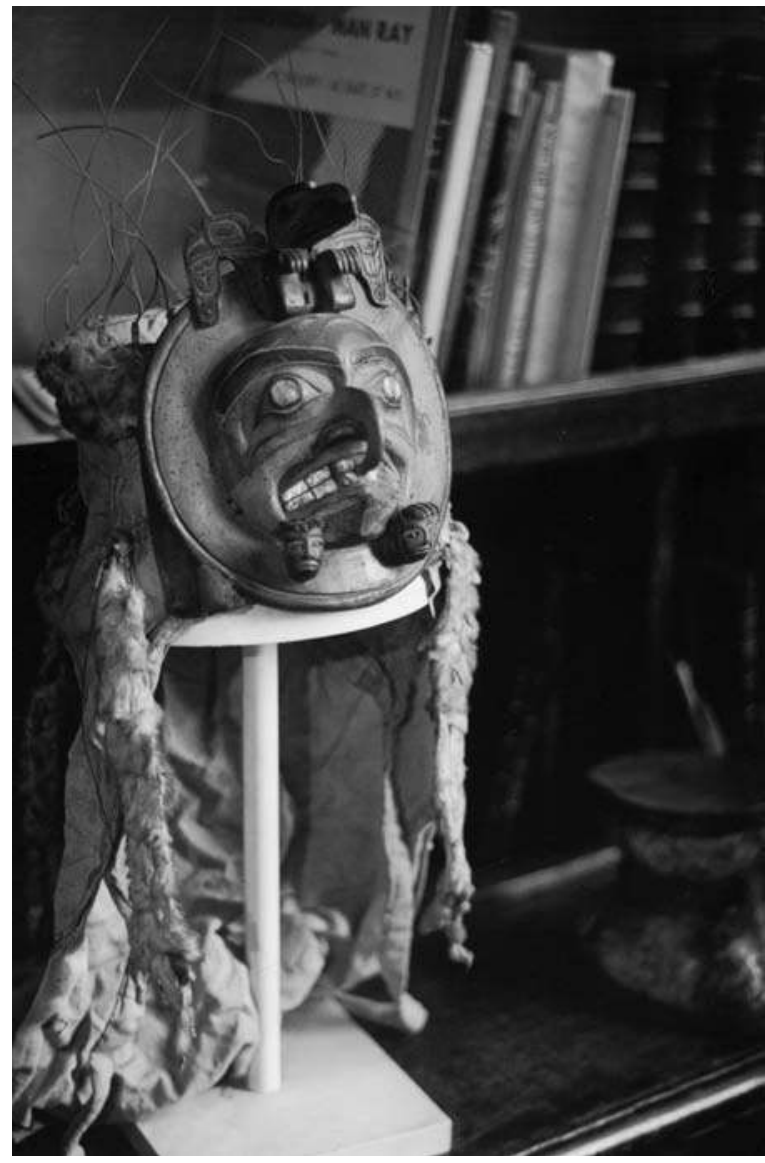

Fig. 7 et $8 \mathrm{Ci}$-dessus et ci-contre : la coiffure dans la bibliothèque des Breton. Elle trouvera sa place définitive sur le bureau (voir fig. 1). Photo Pierre Amrouche, DR.

qui fonde une nouvelle interprétation des objets de la Potlatch Collection.

Élevés au rang d'objets ethnographiques faisant partie d'une collection bien identifiée ${ }^{22}$ - quelles qu'aient été les conditions de la collecte-, ces pièces sont destinées à entrer dans le patrimoine national canadien à une période singulière de l'histoire de la nation. À cette époque, se forge le mythe de la construction d'une identité canadienne homogène indissociable de celui de la disparition des cultures autochtones que l'administration accompagne en prenant des mesures politiques drastiques visant à détruire leurs fondements. Dans ce contexte particulier, tout se passe comme si l'intérêt des politiques rencontrait celui des ethnographes : sont collectés des objets associés à des pratiques rituelles combattues avec force par les missionnaires et les administrateurs comme témoins de sociétés dont il ne restera que de faibles traces, mais dont l'existence ancienne donne une profondeur historique à la nouvelle nation, ce qui justifie l'acte de sauvetage de ces objets témoins ${ }^{23}$. Même si elle est considérée comme faisant partie du patrimoine national, la Potlatch Collection est restée cachée à la vue du public car elle est irrémédiablement marquée du sceau infamant de ses origines (Saunders 1997 : 101; Harkin 2005 : 20). La collection a été conservée dans le silence des réserves : deux pièces ont cependant été montrées au musée de l'Homme à Paris en 1969, à l'occasion de l'exposition Chefs-d'œuvre des arts indiens et esquimaux du Canada, avant d'être présentées comme objets d'art à la galerie nationale du Canada à Ottawa en $1970^{24}$.

\section{L'objet « dépaysé 25 》}

En 1964, André Breton vend Le Cerveau de l'enfant de Giorgio de Chirico, dont la magie avait semble-t-il opéré sur le poète pendant plus de quarante ans (Thévenin 1992). Le temps est venu de s'en séparer, peut-être bien plus en raison d'une désaffection pour le peintre que pour des questions financières (Goutier 1991: 429 note 1; Amrouche 2003 : 108). La disparition de la toile laisse un énorme vide dans l'univers imaginaire de Breton, dont il conviendra de "conjurer l'absence " comme le rappelle fort justement Jean-Jacques Lebel (entretien 2004). Le Cerveau de l'enfant constituait le point focal autour duquel était organisée la mise en espace des œuvres dans l'atelier, dont on a écrit qu'il était un « chantier toujours actif » ou la « véritable fabrique du surréalisme » (La Beaumelle 1991 : 48). Commissaire permanent d'une exposition toujours recommencée, Breton célébrait des œuvres en leur assignant une place de choix, les écartait de son champ de vision quand son regard ne s'y accrochait plus ou encore les déplaçait pour les faire entrer en correspondance avec d'autres objets ${ }^{26}$.

22. Il est intéressant de noter sur les inventaires établis par le Victoria Memorial Museum que les objets identifiés comme potlatch paraphernalia au moment où ils entrent dans le musée deviennent ensuite des specimens.

23. Sur le processus de la formation d'une identité nationale canadienne et la place pour le moins ambiguë occupée par les Premières nations dans les discours de l'élite nationale à travers des manifestations artistiques et culturelles au long des années 1920 et 1930, voir notamment Dawn (2007).

24. Voir Chefs-d'œuvre des arts indiens et esquimaux du Canada, masques $n^{\circ 5} 63$ et 64 . Ces deux masques sont présentés comme « recueillis par D.C. Scott, 1922 ». En réalité, ils font partie d'un ensemble de onze pièces de la Potlatch Collection qui ont été conservées par le surintendant des Affaires indiennes, Duncan Campbell Scott, dans son bureau (Cole 1985 : 254), peut-être à titre de «trophées de guerre ». Scott était un fanatique ; toute sa politique a été inspirée par l'idée de l'assimilation des Indiens par la religion, l'école et le travail (voir Titley 1986).

25. J'emprunte cette expression à Jean Jamin (2004).

26. La première description de l'atelier d'André Breton a été publiée sous la plume d'Alain Jouffroy (1955). 
L'absence de ce tableau, souligne Jean-Michel Goutier, autour duquel semblaient graviter le jour les objets de l'atelier et la nuit les rêves du poète, entraînait obligatoirement la recherche d'un nouvel axe d'aimantation. C'est ainsi que, tournant le dos à la place, au-dessus du lit, précédemment occupée par la célèbre toile de De Chirico, se dressa alors sur la table de travail de l'auteur du Surréalisme et la peinture une statue de Nouvelle-Irlande dite Uli (1991 : 427).

À laquelle s'ajouteront la coiffure kwakwaka'wakw et une figure tolai de Nouvelle-Bretagne, qui elles aussi prendront place sur son bureau, faisant face au fameux " mur » de l'atelier ${ }^{27}$. Pourquoi le remplacement quasi immédiat du tableau par une pièce extra-européenne qui fait système avec deux autres objets dans une nouvelle configuration spatiale? En substituant trois objets " sauvages " à une toile moderne, Breton exclut d'emblée toute hiérarchie entre art occidental et non occidental : les arts océanien et nord-amérindien ont été mis à l'honneur par les surréalistes depuis les années 1920 dans leurs expositions et leurs revues, et évoqués au détour d'une phrase comme dans les entretiens de Breton avec André Parinaud (Breton 1973). Pourquoi la figure uli ? Par l'achat de cette pièce en 1964 à la Foire

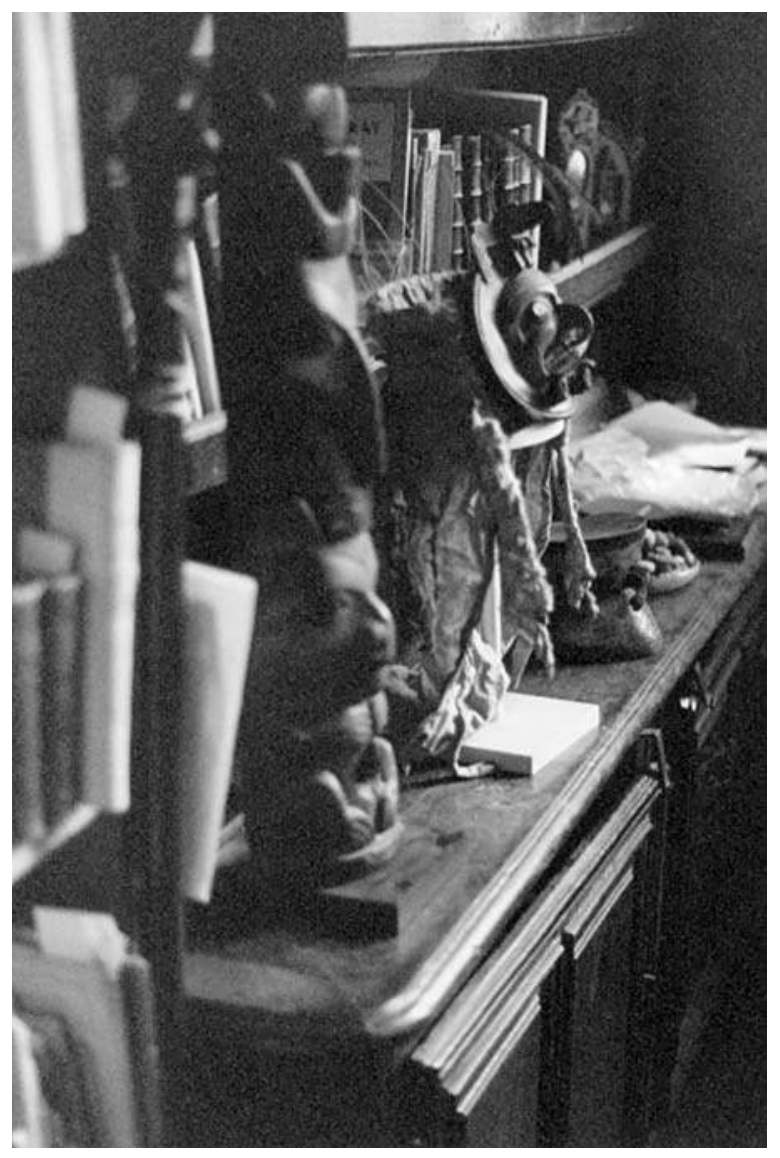

des antiquaires ${ }^{28}$, Breton pouvait satisfaire un désir fort. Il convoitait cet uli depuis la vente Roland Tual de 1930, mais n'avait pu l'acquérir alors. Quelques années plus tard, il lui avait rendu hommage en composant le poème fameux Uli (1948) pour le catalogue de l'exposition Océanie à la galerie Andrée-Olive (Goutier 1991 : 429 ; Amrouche 2003 : 110). Plus qu'à un objet, c'est à un être familier qu'il s'adresse, un " grand dieu " qu'il tutoie comme pour mieux se concilier ses pouvoirs : "Ce grand dieu, écrit-il, qui fait peur [qui] émerveille ${ }^{29}$. " Alors que Breton est au crépuscule de sa vie, " [1]e "passage" du Cerveau de l'enfant à Uli est un acte magique " (Goutier, ibid.), qui permet à la fois de réaffirmer les valeurs qu'il a cru déceler dans l'art océanien d'une part, et de substituer d'autre part un sentiment négatif - celui à l'égard du peintre ingrat à l'endroit des surréalistes - par un sentiment positif engendré par la présence protectrice de l'effigie; ou, pour le dire autrement, à travers cette substitution il s'agissait de "renverser la vapeur poétique ${ }^{30} "$.

L'absence du Cerveau de l'enfant sera compensée par un objet longtemps désiré, auquel s'adjoindra cette coiffure kwakwaka'wakw qui fascine d'emblée Breton. Selon Jean-Jacques Lebel (entretien 2004), témoin avec Joyce Mansour ${ }^{31}$ de la « rencontre » du poète avec l'objet, Breton

27. Le «mur» a été transplanté au centre Georges-Pompidou où il a été montré pour la première fois au public en 2000 . II représentait une « pièce » importante de l'exposition La Révolution surréaliste, organisée en 2002 par Werner Spies. Se reporter aux photographies de l'atelier prises par Gilles Ehrmann en 1998, publiées in 42 rue Fontaine (Ehrmann 2003), et au film réalisé par Fabrice Maze.

28. Communication personnelle de Jean-Michel Goutier, 2 février 2007.

29. Voir Goutier 1991. Le uli devenait un personnage à part entière. De ce fait, le terme devient un nom propre, d'où le $U$ majuscule. La figure uli appartient à la catégorie des malanggan (Nouvelle-Irlande), objets qui représentaient des entités humaines et non humaines. Ils étaient exposés lors de cérémonies funéraires ou initiatiques du même nom. Contrairement aux autres objets malanggan, les uli n'étaient pas détruits à l'issue des cérémonies, mais enveloppés dans des feuilles et accrochés aux chevrons de la maison des hommes pour être réutilisés au cours de nouvelles cérémonies malanggan (Derlon 1991 : 185, 200, 1997 : 139 et suiv. ; Bounoure 1993 : 152 ; Gunn 2000 : 291). Marqués d'une double identité sexuelle, les uli ne représentent pas, comme on l'a longtemps pensé, « des ancêtres dotés du pouvoir et de la force indispensables à un chef de clan » (in Gunn, ibid.), mais font référence à la fois à l'entité lunaire et au mort (Derlon 1991 : 204, 1997 : 123, 2007 : 174). Concevant le dispositif du bureau de son père comme une œuvre à part entière, Aube Elléouët-Breton a fait don de la table de travail et du uli à la bibliothèque Jacques-Doucet, où sont conservés la correspondance et les manuscrits de Breton. La coiffure devait également faire partie de la donation à la bibliothèque.

30. Je dois à Jean-Michel Goutier de m'avoir suggéré l'emploi de cette expression, entretien du 30 janvier 200 ?.

31. Joyce Mansour (1928-1986), amie de Breton, poétesse et nouvelliste surréaliste d'origine égyptienne. 
a eu un " coup d'amour ». Son comportement a trahi l'émotion qu'il a ressentie en la découvrant du regard, puis en la prenant entre ses mains pour en caresser les peaux d'hermine (Lebel, op. cit.). Breton avait déjà été préparé à la rencontre par la description éloquente qu'avait faite la journaliste Paule-Marie Grand dans Le Monde ( 5 juin 1965) de cette " coiffure-masque très précieuse " dont « le masque proprement dit est en bois, rehaussé pour les yeux et les dents, d'incrustations bleutées en nacre d'haliotis [sic] et enrichi de poils de lion de mer et de pendants d'hermine ", termes qui ne pouvaient qu'aiguiser la curiosité de Breton. C'est précisément en des termes proches que les œuvres de la côte nord-ouest étaient décrites dans le milieu surréaliste new-yorkais des années 1940, à l'égal, par exemple, des qualificatifs utilisés par Isabelle Waldberg pour parler de la collection de Claude Lévi-Strauss (1992 : 223). Le mode de connaissance de l'objet s'instaure dans l'immédiateté de l'expérience sensorielle et non dans l'appréhension des qualités formelles de l'œuvre ${ }^{32}$. L'émotion est suscitée par le plaisir tactile de la matière soyeuse de l'hermine et par l'éclat chatoyant de la nacre d'haliotide qui orne les yeux et la bouche du faucon sculpté en haut relief. Outre la séduction immédiate qu'a exercée l'œuvre kwakwaka'wakw sur Breton, celle-ci le transporte dans le monde puissant des chefs chamanes de la côte nord-ouest, comme si le contact de l'objet permettait par imprégnation l'identification "magique " à la personne sociale qui en est le légitime titulaire.

Dans l'atelier de la rue Fontaine, la coiffure occupe une place de choix sur la table de travail (voir fig. 1). Elle fait face à Breton dans une proximité tantôt silencieuse, tantôt animée. À cette "présence " intime sont associées deux autres " présences 33 " fortes qui président et encadrent le dispositif de protection, à savoir le Uli dont on a parlé et une effigie aux bras levés, paumes ouvertes, figure puissamment masculine représentant un esprit dans des rituels d'initiation masculine chez les Tolai de Nouvelle-Bretagne dans le catalogue de la vente André Breton par Calmels-Cohen ${ }^{34}$.

Mais au-delà de ce constat, peut-on se risquer à aller plus loin dans l'analyse de cette nouvelle mise en scène, qui ne peut être envisagée que par rapport à l'accrochage précédent où Le Cerveau de l'enfant constituait un des points d'aimantation de l'univers mental du fondateur du surréalisme? Breton, on le sait, ne s'est jamais véritablement exprimé sur la mise en scène de sa collection (Spies 2007 : 29). Fasciné, cependant, par le jeu des regards, il avait encadré Le Cerveau de l'enfant, représentant un homme aux yeux clos - auquel il avait déjà prêté un regard appuyé dans "L'Almanach surréaliste du demisiècle » (Breton 1950 : 143 ; Goutier 1991 : 429 ; Bounoure 1992 : 70)-, de deux masques à transformation de la côte nord-ouest aux yeux ouverts, dont l'un avait « un regard extraordinairement dur et fixe " comme pour renforcer l'effet hypnotique de la toile ${ }^{35}$. Pour Breton, Le Cerveau est « entre toutes les œuvres plastiques contemporaines celle [qui lui] apparaît la plus chargée de "magie quotidienne" "; en outre, elle " est douée [...] d'un pouvoir de choc exceptionnel » (1970 : 38-39). Il précise :

Le tableau chez moi ayant pris place entre deux masques «à transformation » de Colombie-Britannique auxquels un dispositif de ficelles permet à volonté d'ouvrir et de fermer les yeux [...], il m'est difficile de savoir si le besoin de prêter un regard à ce visage (exsangue et sans âme) a été surdéterminé par le voisinage des masques ou si, au contraire, c'est le besoin encore subconscient qui m'a incité à les suspendre de part et d'autre de lui (1970: 42, note 2).

Si, comme le remarque Goutier (1991), « le jeu des métamorphoses, entre Uli et Le Cerveau de l'enfant, passe par le balancier du regard ", on comprend que Breton ait opéré la substitution en transformant un non-regard en un regard pénétrant-rappelons le vers de Breton à propos d'Uli : «Tu nous reluques d'un fond de coquillage »-, regard qui s'exprime par des yeux formés de coupelles de nacre avec des pupilles en opercule de coquillage $\left(\right.$ turbo $\left.^{36}\right)$ et s'exacerbe au contact de celui

32. Voir notamment Derlon et Jeudy-Ballini (2005 : 148-149).

33. Le mot «présence » est emprunté à Alain Jouffroy. Selon lui, Breton «ne rassemblait pas des objets, mais reconnaissait des présences », in Pataud Célérier 2003 : 80.

34. C'est ce que montre la comparaison entre la photographie prise en 1965 par Gisèle Freund in André Breton. La beauté convulsive (1991: 82-83), le film de Fabrize Maze et les clichés de Gilles Ehrmann, datant de 1998, qui témoignent de l'état dernier de l'atelier tel que la mort de Breton l'avait figé. Je dois à Jean-Michel Goutier les informations concernant l'année précise des clichés de Freund et d'Ehrmann. Pour l'effigie tolai, voir André Breton, 42 rue Fontaine. Arts primitifs 2003 : 94 ; Corbin $1998: 266$.

35. À propos du masque accroché à gauche de la toile, Breton écrit : « C'est ce masque haida [...] dont le regard extraordinairement dur et fixe peut se recouvrir de paupières turquoises 》 (1950: 41). Rappelons les mots de Jouffroy à propos du personnage du tableau de De Chirico: «L'immobilité, l'aveuglement, la pesanteur, l'anonymat de la figure, véritable sphinx ininterprétable, hypnotisent vraiment le spectateur » (1955: 39). Une photographie de Sabine Weiss, publiée notamment dans l'article de Jouffroy (ibid. : 35) puis dans le catalogue Sculptures (2000 : 288), montre le mur de la chambre, où l'on reconnaîtra, outre les deux masques, un bâton de harangue sculpté et une cuiller en corne au manche ouvragé provenant également de la côte nord-ouest, le Portrait de Washington de Marcel Duchamp et une Tête de femme de Pablo Picasso.

36. Voir description d'Uli in André Breton. 42 rue Fontaine. Arts primitifs 2003 : 108. 


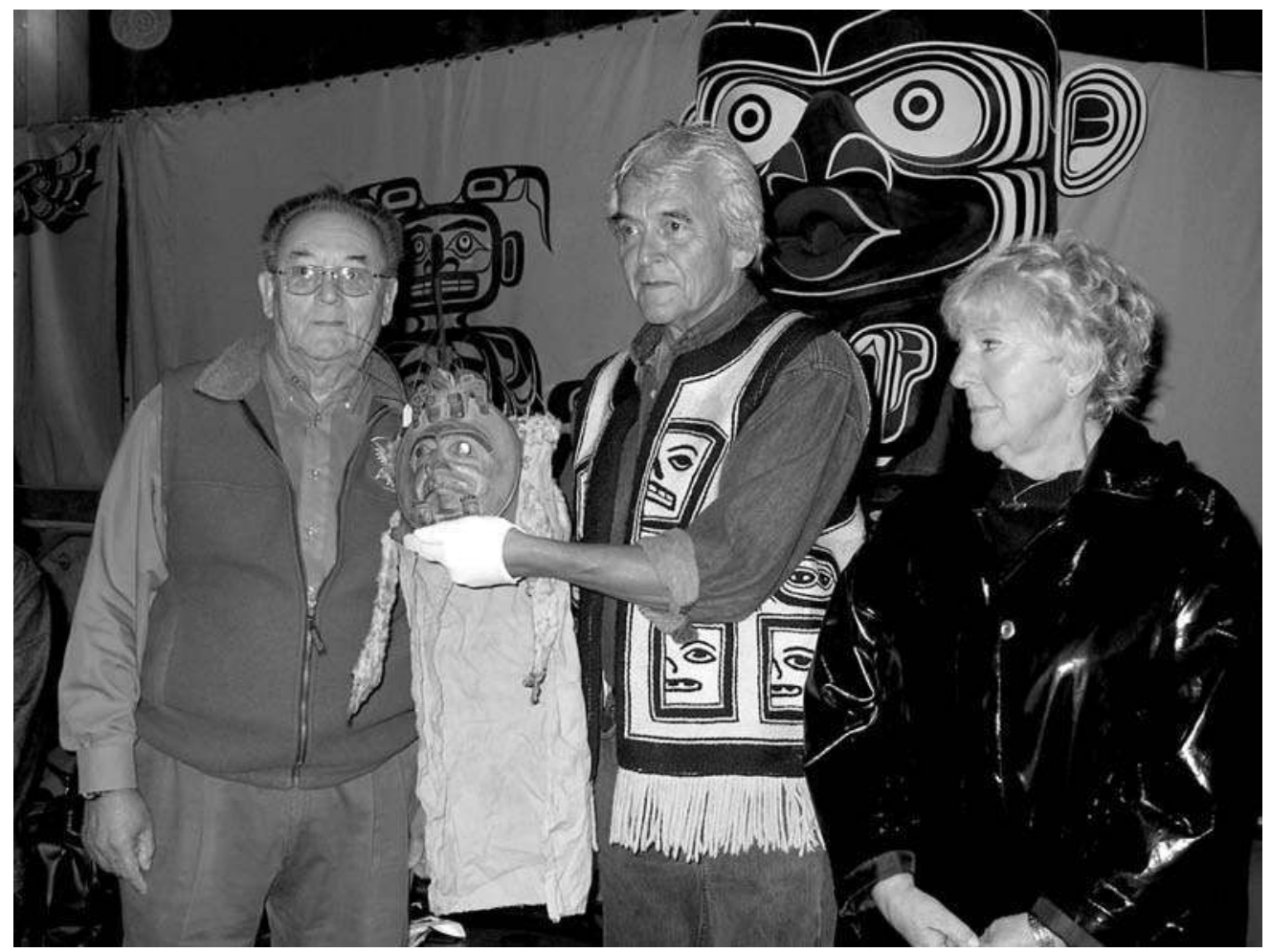

Fig. 9 Restitution de la coiffure dans la maison cérémonielle d'Alert Bay. Au centre, Bill Cranmer, à gauche Don Assu, à droite Aube Elléouët-Breton, septembre 2003. Photo Pierre Amrouche, DR.

quasiment fixe et aveuglant que renvoie le reflet chatoyant de l'haliotide des yeux du faucon de la coiffure.

Cette tentative de saisir l'imagination poétique de Breton est certainement une infraction malheureuse car ce dernier est « le seul à percevoir les forces d'attraction [et à] être capable aussi de les susciter" (La Beaumelle $1991: 61$ ). C'est son regard unificateur « qui travaille avec des associations iconographiques ou formelles " et établit des analogies entre des choses fort différentes, des formes éloignées à la fois dans l'espace et le temps (Spies 2007 : 31). Les rapports entre peintures et objets de toute origine " [...] s'explicitaient pour Breton lui-même en relations de proximité ou de distance dans la disposition topographique des choses " (Bounoure 1992 : 70 ; voir aussi Dufour 1994).

La signification propre d'une œuvre n'est-elle pas, non pas celle qu'on croit lui donner, mais celle qu'elle est susceptible de prendre par rapport à ce qui l'entoure (Breton 1924 [1988] : 198)?

En percevant de manière aiguë des résonances entre ces choses, Breton se donne le rôle d'un observateur magicien qui « dépayse » les œuvres, les conduisant loin de leurs terres d'origine pour les re-territorialiser dans un champ de signification construit par une pratique créatrice qui pourrait s'apparenter à une forme de magie :
"La magie, souligne-t-il, est une pratique fournissant un moyen d'agir sur un élément de l'univers en utilisant des correspondances analogiques que cet élément possède avec tout autre élément de l'univers " (1970: 42-43). Breton envisage l'art non pas en termes d'esthétique et de beauté, mais en termes de force suggestive ou de système d'action dont l'efficacité se mesure à l'effet qu'il produit sur les choses et les hommes (Gell 1998). Il considérait que, pour accéder à la condition d'œuvres, les objets doivent être dotés d'une charge ou, dirait-on aujourd'hui, d'une intentionnalité agissante dont l'intensité augmente ou se transforme dans la proximité de leur contact. Tout se passe comme si Breton avait restitué à la coiffure une "force » qu'elle avait perdue comme artefact sommeillant dans les réserves d'un musée, tout en lui attribuant des intentionnalités qui ressortissent à son propre système magico-poétique.

\section{L'objet patrimonial}

Un moment exceptionnel dans la vie de la coiffure fut certainement sa réintégration dans sa communauté d'origine kwakwaka'wakw à l'issue d'une cérémonie de restitution organisée dans la Big House d'Alert Bay, qui a été ouverte par un banquet. Discours, danses, 
chants et distribution de cadeaux en ont ponctué les différents moments. Restituée par une personne individuelle et non une institution, la coiffure a été traitée différemment par rapport aux quelque quatre cents objets déjà rendus par le musée canadien des Civilisations, le Royal Ontario Museum et le National Museum of the American Indian. C'est bien autour d'un objet unique qu'a été organisée la cérémonie : la coiffure a été exhibée par des danseurs alors que les pièces préalablement restituées par les institutions muséales avaient été présentées aux membres des communautés kwakwaka'wakw soit dans le musée local lui-même, le jour de son inauguration, soit dans la Big House où elles étaient exposées sur des tables. Outre que le retour de la coiffure a conduit les responsables du musée et les Anciens à inventer un rituel $a d$ hoc, il n'en demeure pas moins qu'ils ont voulu saluer la démarche exceptionnelle d'une personne - la fille d'un collectionneur privé inconnu d'eux - qui a empêché que la coiffure ne leur échappe à jamais ${ }^{37}$. Aube Elléouët-Breton a été honorée du nom d'uma, ou " femme noble ", en reconnaissance de la générosité de son geste; elle a été ensuite invitée à danser la Ladies' Dance, prérogative dont se prévalent les femmes de haut rang ${ }^{38}$.

Placée dans une caisse en bois, la coiffure est introduite dans la Big House au son du tambour par Aube Elléouët-Breton et sa fille Oona, assistées de la directrice et d'un membre du conseil d'administration du musée, pour être déposée devant les trois chefs kwa kwaka'wakw qui président la cérémonie (Bill Cranmer, Adam Dick et Don Assu). La coiffure est ensuite montrée au public par Bill Cranmer, chef nimpkish et maître de cérémonie ${ }^{39}$, puis dansée autour de la Big House par chacun des chefs, suivis par une longue procession formée par les membres de la communauté et des danseurs du T'salala Cultural Group ${ }^{4}$. La coiffure est présentée à hauteur de poitrine par les chefs, dont les mains sont gantées de blanc comme le sont celles des conservateurs de musée quand ils manipulent des objets. Elle n'est pas portée sur la tête comme dans une cérémonie " traditionnelle », ce qui aurait été considéré comme le détournement d'un privilège ancestral étant donné que l'identité de son vrai propriétaire n'est pas connue.

La cérémonie a permis de produire une coalescence ou, si l'on préfère, une rencontre entre deux registres de signification à la fois antagonistes et complémentaires au vu de la situation présente. La coiffure est réintroduite rituellement au sein d'une cérémonie inédite créée pour l'occasion, qui renvoie au contexte rituel d'origine de l'objet et qui respecte le protocole cérémoniel, l'objet n'étant exhibé que par des chefs détenteurs du droit à ce rituel. Elle est aussi considérée comme une pièce de musée, ce qu'elle est définitivement. Nul ne cherche à réinstaurer dans l'objet une authenticité désormais perdue. Dans le cadre du musée local, elle a pris le statut d'artefact : elle a été inventoriée et, après restauration, sera exposée dans la galerie principale de l'établissement, aux côtés de ses compagnons d'infortune auxquels elle a faussé compagnie pendant quelque quatre-vingts ans. L'exemple de ce qu'on pourrait appeler le dernier temps de l'existence de la Potlatch Collection montre bien qu'un objet, une fois sorti de son contexte d'origine, ne peut généralement pas retrouver son statut initial même dans le cadre d'une restitution : outre qu'il est rendu non à un individu mais à une collectivité institutionnelle, il est rarement réutilisé dans les rituels; il acquiert le plus souvent une valeur radicalement nouvelle.

Au U'Mista Cultural Centre, l'ensemble des objets restitués est exposé sans que des distinctions soient opérées entre eux, tous également témoins de la répression gouvernementale visant les pratiques cérémonielles traditionnelles. Le parti pris muséographique a consisté à confronter le visiteur à une procession d'objets disposés sur le pourtour de la galerie-maison cérémonielle, dans une semi-obscurité, de telle sorte que les rôles soient inversés : c'est le visiteur qui est soumis au regard des masques, des cuivres et autres regalia. Au centre de la galerie, sur une estrade rectangulaire, les imposants masques humsumtl font masse et saisissent l'observateur ${ }^{41}$. Le visiteur est invité à se déplacer autour de la galerie en respectant le tracé du parcours de tout danseur de masque, qui s'effectue

37. Outre le fait que j'avais identifié la pièce, la restitution n'a été possible que grâce à la généreuse sympathie d'Aube Elléouët-Breton. La correspondance échangée avec le U'Mista Cultural Centre montre que le personnel du musée a été surpris par son initiative.

38. Le terme fait référence aux qualités morales dont doit faire preuve une femme de haut rang chez les Kwakwaka'wakw, qui reconnaissent à Aube Elléouët-Breton la dignité d'une femme noble.

39. Bill Cranmer est le fils de Dan Cranmer, le chef qui avait organisé le potlatch de 1921 à la suite duquel les objets cérémoniels ont été confisqués. Il est présent en sa qualité de chef élu de la bande nimpkish, de chef héréditaire et de président du conseil d'administration du U'Mista Cultural Centre (communication d'Andrea Sanborn, janvier 2007).

40. Groupe de danseurs qui se produit dans la Big House à l'occasion de performances ou spectacles organisés pour les touristes qui visitent la communauté d'Alert Bay, qui accueille des bateaux de croisière. II se produit également dans les musées.

41. Les masques humsumt/ représentent les assistants de l'esprit cannibale dans le rituel dit hamaca. Ils figurent des oiseaux mythologiques et peuvent atteindre un mètre cinquante de long. 


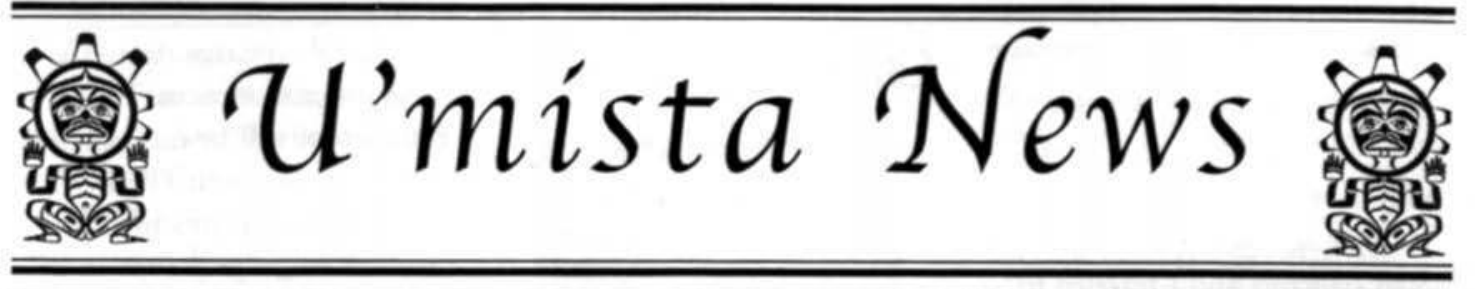

\section{Yaxwiwe' Returning Home!}

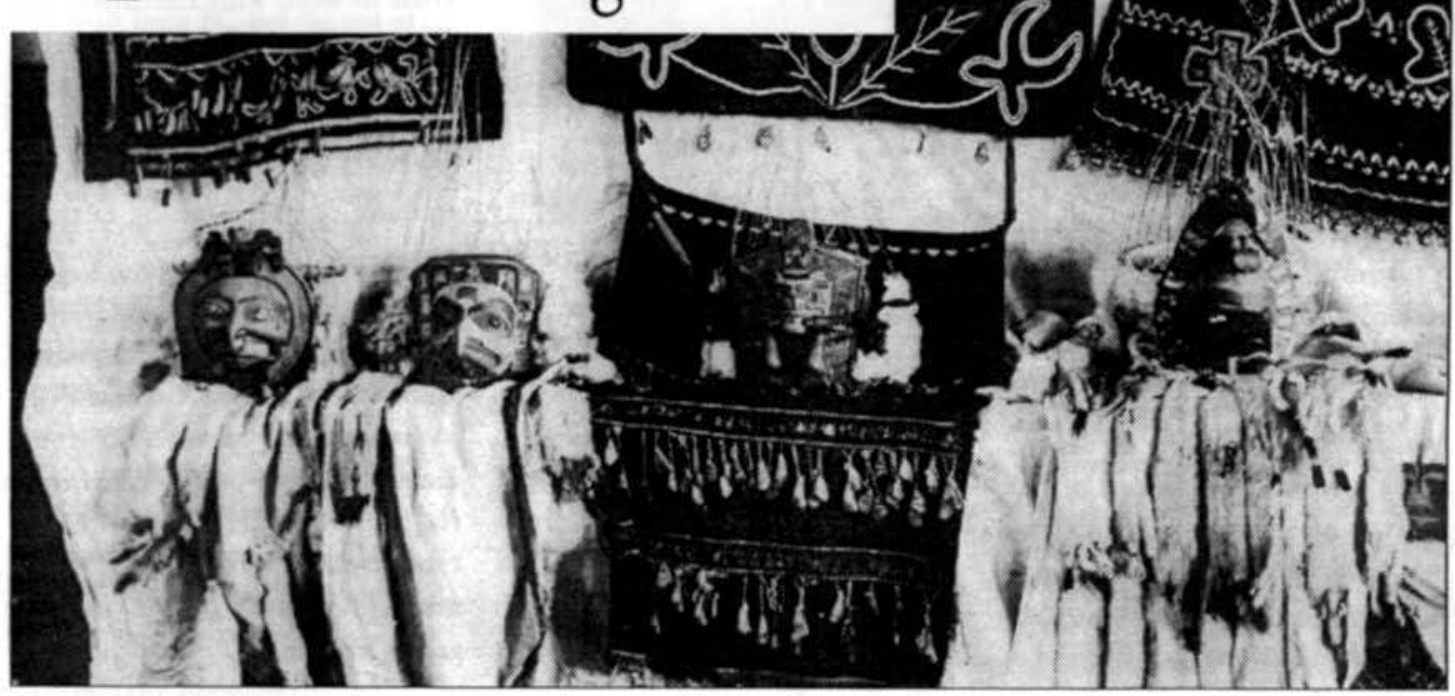

Surrendered Potlatch Regalia, consisting of yaxwiwe' (peace dance headdresses) and aprons, photographed in the Parish Hall, Alert Bay, B.C. 1922. The yaxwiwe' is on the lower bench, far left.

Photograph Courtesy of the Royal British Columbia Museum PN 9923

AS WE MENTIONED briefly in the last newsletter, one of the missing pieces from the Potlatch Collection has been found! The piece was one of those deaccessioned and we had completely lost track of its location. Through the efforts of Dr. Marie Mauzé and the generosity of Mme Aube Elléouët we are looking forward to welcoming another piece of the Potlatch Collection home. Dr. Mauze, Mrs. Elléouët and $\mathrm{Mr}$. Cyrille Cohen will be travelling to $\mathrm{BC}$ in mid-September and will arrive in Alert Bay on the 20th to return the yaxwiwe' to the Kwakwak a'wakw.

A yaxwiwe' (peace dance headdress) is worn in the Tta'sala, the second major part of the potlatch.
The word Tta'sala can not be translated into English. It may have been the name for this dance among one of the Northern tribes from whom we obtained this dance. The Tsimshian, Tlinget and Haida all used this dance, they call it the Peace Dance or Chief's Dance. Headdresses also originated from the Northern tribes and consist of a carved wooden plaque, called a frontlet, depicting a crest animal. The frontlet is attached to a band of cedar bark lined with swan down and feathers. A crown of baleen or sea lion whiskers give the headdress height. The train or cape consists of ermine and rabbit and give the yaxwiwe' its elegance.

Contimued on page 6

Fig. 10 Première page du bulletin U'Mista News annonçant la restitution de le la coiffure par Aube Aube Elléouët-Breton et sa fille Oona. @ U'Mista Cultural Centre. 
dans le sens des aiguilles d'une montre. Dans le même temps, en passant devant différents types de masques disposés en fonction des séquences rituelles successives intervenant dans un potlatch, il accomplit, sans en avoir conscience, le cycle cérémoniel dans sa tota-

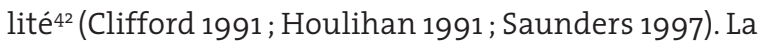
disposition des masques communique en quelque sorte un message codé que seuls les Kwakwaka'wakw connaisseurs de leur culture peuvent saisir immédiatement car aucune indication ne signale cette répétition du cycle ancestral. Si la muséographie donne à voir la richesse de la culture matérielle kwakwaka'wakw par la grande variété des pièces sculptées, elle est surtout destinée à provoquer un effet de sidération sur le spectateur, ou du moins à susciter une réflexion sur la condition des peuples autochtones au moment où le Canada s'affirmait comme nation. Le dispositif narratif est complété par des panneaux et des cartels reproduisant des documents, notamment des correspondances entre agents locaux et surintendant du département des Affaires indiennes sur l'observance et la non-observance de la loi anti-potlatch et sur les moyens de réprimer cette dernière, des pétitions de chefs protestant contre la suppression du potlatch et des récits de témoins de l'époque de la confiscation et des emprisonnements. Voix officielles, voix indiennes sont confrontées pour dire chacune leur vérité, mais la présence à la fois muette et imposante des objets apporte la preuve matérielle de la politique répressive du gouvernement canadien et de la résistance à cette oppression, provoquant ainsi l'empathie ou l'adhésion du visiteur. Dans ce contexte, l'objet témoin met à mal la parole gouvernementale et renforce le discours de survivance kwakwaka'wakw.

Si toute forme d'exposition est un acte d'interprétation, on mesure le contraste entre l'exposition de 1922 dans la salle paroissiale, où les objets étaient traités sans ménagement, ce qui s'apparentait à une forme de désacralisation, et l'exposition permanente au U'Mista Cultural Centre où ils apparaissent comme les supports d'un discours politique à destination d'un public non autochtone plutôt que local (voir aussi Saunders 1997 : 109-110). Les modalités d'exposition se donnent à voir comme la métaphore de deux situations politiques et culturelles diamétralement opposées : celle d'un peuple

42. Au Kwagiulth Museum, la muséographie met l'accent sur le patrimoine symbolique des chefs et des familles nobles - les objets sont regroupés dans des vitrines en fonction du nom de chaque propriétaire. Au U'Mista Cultural Centre, l'origine familiale et tribale des objets est oblitérée (voir Mauzé 1999a : 426-427). vaincu sous les coups de boutoir du gouvernement canadien et celle d'un peuple qui pose un regard accusateur sur l'histoire de la colonisation et de la domination canadienne tout en négociant son identité au sein de la société globale.

À l'instigation du National Museum d'Ottawa, les Kwakwaka'wakw ont fait leur l'idée que la Potlatch Collection faisait partie de leur patrimoine culturel, ce qui leur permet de revendiquer dans le cadre de cette économie politique d'exposition (Kirshenblatt-Gimblett 1991) une culture qui leur est propre et une identité « nationale » vis-à-vis des non-autochtones, même si l'on constate l'incapacité récurrente des communautés à faire cause commune dans leurs revendications (Saunders 1997; Mauzé 1999a : 426; Harkin 2005).

Ce travail d'investigation permet de saisir les différents usages et valeurs qui ont été attribués à la coiffure au cours de son existence. Elle partage avec les objets de la Potlatch Collection une communauté de destin, mais ne s'en singularise pas moins en ce que sa vie est liée à des déterminations qui lui sont extérieures. C'est l'interaction des destinées particulières qui crée le destin de l'objet. Peut-on penser que la restitution de la coiffure aux Kwakwaka'wakw par Aube Elléouët-Breton et sa nouvelle vie au musée d'Alert Bay vont mettre un point final à ses aventures ?

mots clés / keywords : côte nord-ouest // North-West coast - Amérique du Nord (Canada) // North America (Canada) - kwakiutl (kwakwaka'wakw) // Kwakiutl (Kwakwaka'wakw) - collecte // collecting • collection // collection • potlatch // potlatch • surréalisme // surrealism • restitution // repatriation • objet // object • Premières nations // First Nations.

Laboratoire d'anthropologie sociale mauze@ehess.fr

\footnotetext{
* Je tiens à remercier chaleureusement les amis grâce auxquels la restitution a été possible, et tout particulièrement Aube Elléouet-Breton et sa fille Oona. Pierre Amrouche et Cyrille Cohen ont soutenu cette initiative. Mes remerciements vont également au chef Bill Cranmer, président du U'Mista Cultural Centre, au chef Don Assu, président de la Nuyumbalees Society (Kwagiulth Museum and Cultural Centre), et à Andrea Sanborn, directrice du U'Mista Cultural Centre qui ont accueilli une petite délégation française dans la maison cérémonielle d'Alert Bay le 23 septembre 2003. Outre les amis cités, Aube avait réuni autour d'elle Laurence Calmels, Marcel et Clo Fliess, Jean-Claude Georges, Stéphane Vauclain pour la cérémonie de restitution. À l'automne 2005, j'ai entrepris des recherches dans les archives du U'Mista Cultural Centre grâce à une bourse de recherche du gouvernement canadien et un soutien financier du groupe de recherche «Anthropologie, objets et esthétiques » (GDR 2156).
} 


\section{Bibliographie}

Ames, Michael

1992 Cannibal Tours and Glass Boxes:

The Anthropology of Museums. Vancouver, University of British Columbia Press.

1994 « Cannibal tours, glass boxes and the politics of interpretation », in Susan M. Pearce [éd.], Interpreting Objects and Collections. Londres et New York, Routledge : 98-106.

\section{AmRouche, Pierre}

2003 « Les arts primitifs dans la collection Breton », in André Breton, 42, rue Fontaine. Arts primitifs. Paris, Calmels-Cohen : 14-19.

ApPadurai, Arjun

1986 The Social Life of Things. Commodities in Cultural Perspectives. Cambridge, Cambridge University Press.

\section{Bethenod, Martin}

2003 Jacques Kerchache, Portraits croisés. Paris, Gallimard et musée du quai Branly.

\section{Bonnot, Thierry}

2004 «Itinéraire d'une bouteille de cidre», L'Homme 170 : 139-163.

\section{BOUNOURE, Vincent}

1992 « Note sur les arts sauvages en réponse à Pierre Amrouche », L'Autre 4 : 65-76.

1993 Visions d'Océanie. Paris, Dapper.

\section{BRACKEN, Christopher}

1997 The Potlatch Papers. A Colonial Case History. Chicago, University of Chicago Press.

BRETON, André

1924 [1988] «La confession dédaigneuse », in Les Pas perdus (Euvres complètes, vol. I). Paris, Gallimard [ «Bibliothèque de la Pléiade »] : 193-202.

1950 « Notes sur les masques à transformation de la côte nord-ouest de l'Amérique », Neuf 1 , juin : 37-41.

1970 Perspective cavalière [lettre à Robert Amadou, 1953]. Paris, Gallimard : 38-45.

1973 Entretiens avec André Parinaud. 19131952. Paris, Gallimard [《Le point du jour 》).

Breton, André et PÉret, Benjamin

1950 «Almanach surréaliste du demi-siècle », La Nef, numéro spécial mars-avril.

\section{CARPENTER, Edmund}

1975 «Collecting Northwest Coast Art », in Bill Holm et Bill Reid, Indian Art of the Northwest Coast. Houston, Institute for the Arts, Rice University : 9-27.
2005 Two Essays: Chief \& Greed. North Andover, Persimmon Press.

\section{Clifford, James}

1991 «Four Northwest Coast Museums: Travel Reflections », in Ivan Karp et Steven D. Lavine (éd.), Exhibiting Cultures: The Poetics and Politics of Museum Display. Washington, Smithsonian Institution Press : 212-254.

\section{Cole, Douglas}

1985 Captured Heritage. The Scramble for Northwest Coast Artifacts. Vancouver, Douglas \& Mclntyre.

\section{Cole, Douglas et CHAikin, Ira}

1990 An Iron Hand upon the People. The Law against the Potlatch on the Northwest Coast. Vancouver, Douglas \& McIntyre-Seattle, University of Washington Press.

\section{CORBIN, George A.}

1998 « La Nouvelle-Bretagne orientale. Les peuples baining, sulka et tolai », in Arts des mers du Sud. Collections du musée BarbierMueller. Paris, Adam Biro : 256-26?.

\section{DaWn, Leslie}

2007 National Visions, National Blindness. Canadian Art and Identities in the 1920s. Vancouver, UBC Press.

\section{DERLON, Brigitte}

1991 «L'objet malanggan dans les anciens rites funéraires de Nouvelle-Irlande », Res 19-20: 179-210.

1997 De mémoire et d'oubli. Anthropologie des objets malanggan de Nouvelle-Irlande. Paris, CNRS-Maison des sciences de l'Homme.

2007 «Figures uli », in Michael Gunn et Philippe Peltier (éd.)., Nouvelle-Irlande. Arts du Pacifique Sud. Paris, musée du quai Branly-5 Continents : 174.

Derlon, Brigitte et JeUdy-Balini, Monique

2005 «De l'émotion comme mode de connaissance », in Michèle Coquet, Brigitte Derlon et Monique Jeudy-Ballini (éd.), Les Cultures à l'œuvre. Rencontres en art. Paris, Biro Éditeur : 147-166.

\section{Dufour, Bernard}

1994 Des collectionneurs tel André Breton. Cognac, Fata Morgana.

\section{EHRMANN, Gilles}

200342 rue Fontaine. L'atelier d'André Breton, texte de Julien Gracq, photographies de Gilles Ehrmann. Paris, Adam Biro.

FEEST, Christian

1998 « Transformation of a mask: Confidential intelligence from the lifeway of things $\gg$,

Baessler-Archiv, Neue Folge, vol. XLVI : 255-293.

FORCE, Roland

1999 The Heye and the Mighty, Politics and the Museum of the American Indian. Honolulu, Mechas Press.

GeLL, Alfred

1998 Art and Agency. An Anthropological Theory. Oxford, Clarendon Press.

\section{Goutier, Jean-Michel}

1991 «Au regard d'Uli », in André Breton. La beauté convulsive. Paris, musée national d'Art moderne-centre Georges-Pompidou : 427-431.

2003 «Étant donné... », in André Breton, 42 rue Fontaine. Arts primitifs. Paris, Calmels-Cohen : 9-13.

GunN, Michael

2000 «Statue de Nouvelle-Irlande », in Jacques Kerchache, avec Vincent Boulouré (éd.], Sculptures. Afrique Asie Océanie Amériques. Paris, Réunion des musées nationaux-musée du quai Branly : 290-291.

\section{HaLliday, William}

1935 Potlatch and Totem and the Recollections of an Indian Agent. Londres et Toronto, J.M. Dent $\&$ Sons.

\section{HARKIn, Michael}

2005 « Object lessons. The question of cultural property in the age of repatriation », Journal de la Société des américanistes 9(2) : 9-29.

\section{HouliHAN, Patrick}

1991 « The poetic image and native American art », in Ivan Karp et Steven D. Lavine [éd.], Exhibiting Cultures: The Poetics and Politics of Museum Display. Washington, Smithsonian Institution Press : 205-211.

HunT, George

1924 « The History of 21 Coppers », in Boas Collection. Philadelphia, American Philosophical Society (manuscrit).

InGLIS, Stephen

1990 « "Good Lord Man, this Stuff is Crude:" Beneath the Patina of Canadian Folk Art », in Boyd White et Lynn M. Hart (éd.), Living Traditions in Art: First International Symposium. Montréal, McGill University Press : 92-100. 


\section{ÉTUDES ET ESSAIS}

JAMIN, Jean

2004 « La règle de la boîte de conserve », L'Homme 179 : $7-10$.

\section{JoUfFroy, Alain}

1955 « La collection André Breton », L'EFil 10 : 32-39.

Kerchache, Jacques, Boulouré, Vincent (éd.)

2000 Sculptures. Afrique, Asie, Océanie, Amériques. Paris, Réunion des musées nationaux-musée du quai Branly.

KeRchache, Jacques et De RouX, Emmanuel

«Une centaine de chefs-d'œuvre "primitifs" vont entrer au Louvre », Le Monde, 15 juillet 1998.

\section{KiRSHENBLATT-GimbLETT, Barbara}

1991 « Objects of ethnography », in Ivan Karp et Stephen D. Lavine (éd.), Exhibiting Cultures. The Poetics and Poetics of Museum Display. Washington, Smithsonian Institution Press : 386-443.

1998 Destination Culture: Tourism, Museums, and Heritage. Berkeley, University of California Press.

\section{KOPYTOFF, Igor}

1986 « The cultural biography of things: commoditization as process 》, in Arjun Appadurai (éd.), The Social Life of Things. Commodities in Cultural Perspectives.

Cambridge, Cambridge University Press : 64-94.

\section{La Beaumelle, Agnès de} 1991 «Le grand atelier », in André Breton. La beauté convulsive. Paris, musée national d'Art moderne-centre Georges-Pompidou : 48-63.

\section{LAFORET, Andrea}

1993 « Time and the Grand Hall of the Canadian Museum of Civilization », Museum Anthropology $17(1): 22-32$

LÉvi-Strauss, Claude

1983 Le Regard éloigné, Paris, Plon.

1988 De près et de loin, Paris, Odile Jacob.

Mauzé, Marie

1992 «Exhibiting one's culture: two case studies », European Review of Native American Studies 6[1] : 27-30.

1995 «Potlatching as ever », European Review of Native American Studies 9(2) : 25-31. 1999a «Un patrimoine, deux musées : la restitution de la Potlatch Collection 》, Ethnologie française 29(3) : 419-430.

$1999 b$ « L'éclat de l'haliotide. De la conception du beau dans les sociétés de la côte nordouest », Terrain 32 : 83-98.

2000 «Sculpture kwakwaka'wakw. Pilier de maison gwasila » : 351-355; « Sculpture nisga'a. Masque frontal » : 360-361; «Sculpture kwakwaka'wakw. Masque à transformation » : 362-365; «Sculpture tlingit. Heaume » : 366-369, in Jacques Kerchache (éd.), Sculptures. Afrique, Asie, Océanie, Amériques. Paris, Réunion des musées nationaux - musée du quai Branly.

2004a «Quand une coiffure cérémonielle kwakwaka'wakw retrouve sa communauté d'origine : histoire d'une restitution », Art tribal 5 : 112-11?.

$2004 b$ « Aux invisibles frontières du songe et du réel. Lévi-Strauss et les surréalistes : la "découverte" de l'art de la côte nord-ouest », Cahier de l'Herne 82, Michel Izard (éd.), Claude Lévi-Strauss : 152-161.

2006 《Des surréalistes en exil », Collection Robert Lebel. Paris, Calmels-Cohen : 17-29.

MAZE, Fabrice

2005 DVD André Breton. Paris, Seven Doc-Aube Elléouët-Breton.

\section{PATAud CÉlerier, Philippe}

2003 «André Breton, 42, rue Fontaine :

le cadavre-exquis-boira-t-il encore-

le vin-nouveau », Art Tribal 4 : 80-85.

Rousselot, Jean-Loup

2000 «Sculpture yup'ik» : 433-436; «Sculpture yup'ik »: 436-438 in Jacques Kerchache (éd.), Sculptures. Afrique, Asie, Océanie, Amériques. Paris, Réunion des musées nationaux - musée du quai Branly.

\section{SAUNDERS, Barbara}

1997 « Contested Ethnie in two Kwakawaka'wakw museums », in Jeremy MacClancy (éd.), Contesting Art. Art, Politics and Identity. Oxford, Berg : 85-130.

SAVARD, Dan

2005 «Changing images. Photographic collections of First Peoples of the Pacific Northwest held in the Royal British Columbia Museum, 1860-1920 », BC Studies 145 : 55-96.

SCHAEFFER, Jean-Marie 2004 « Objets esthétiques 》, L'Homme 170 : 22-45.

\section{SEWID-SMITH, Daisy}

1979 Prosecution or Persecution. [Cape Mudge, British Columbia], Nu-yum-balees Society.

SPIES, Werner

2007 « "Le mur" d'André Breton », in L'EFil et le mot. Paris, Christian Bourgois : 27-52.

SPIEs, Werner (éd.)

2002 La Révolution surréaliste. Paris, centre Georges-Pompidou.

THÉVEnin, Paule

1992 «Les énigmes du Cerveau de l'enfant », in André Breton. La beauté convulsive.

Paris, musée national d'Art moderne-centre Georges-Pompidou : 101-105.

Titley, Brian

1986 A Narrow Vision. Duncan Campbell Scott and the Administration on Indian Affairs in Canada. Vancouver, University of British Columbia Press.

\section{TownsEnd-GAULT Charlotte}

199? «Art, argument and anger on the Northwest Coast », in Jeremy MacClancy (éd.), Contesting Art. Art, Politics and Identity. Oxford, Berg : 131-164.

U'Mista Cultural Centre Archives

U'Mista News, hiver 2002 ; été 2003

WALDBERG, Patrick et Isabelle 1992 Un amour acéphale. Correspondance 1940-1949, Michel Waldberg (éd.), Paris, La Différence.

\section{Wallace, Kevin}

1960 «A Reporter at large. Slim-Shin's monument », New Yorker 36, 19 novembre : 104-112.

\section{CATAlogues d'Exposition}

1969 Chefs-d'œuvre des arts indiens et esquimaux du Canada. Paris, Société des amis du musée de l'Homme.

1991 André Breton. La Beauté convulsive, Paris, Centre Georges-Pompidou-IRCAM.

2003 André Breton, 42, rue Fontaine. Arts primitifs. Paris, Calmels-Cohen.

\section{ENTRETIEN}

21 avril 2004, entretien de l'auteur avec Jean-Jacques Lebel, non publié. 


\section{Biographie sommaire de la coiffe}

1922 Confiscation de la Potlatch Collection

1922-1926 Coiffure en possession de Donald Angermann. Coiffure vendue par $M^{\text {me }}$ Angermann à George Heye en 1926

1926-1957 Fait partie des collections de l'American Museum of the American Indian

1957 Edward Primus, galeriste. La coiffure est retirée de l'inventaire du musée
1957-1964 période imprécise

1964-1965 Jacques Kerchache. Exposée en 1964 et en 1965 dans sa galerie rue de Seine

1965-2003 André Breton. Atelier rue Fontaine

2003 U'Mista Cultural Centre. Don par la fille et petite-fille d'André Breton

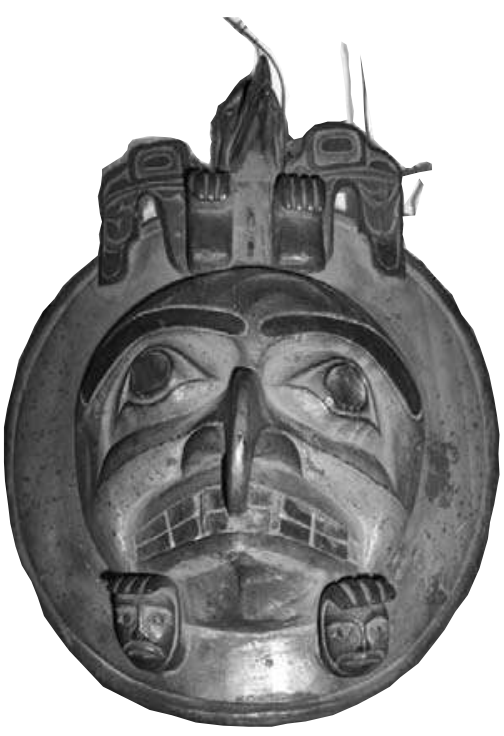

\section{Résumé / Abstract}

Marie Mauzé, Trois destinées, un destin. Biographie d'une coiffure kwakwaka'wakw. - Une coiffure cérémonielle faisant partie de la collection du poète surréaliste André Breton a été restituée aux Kwakwaka'wakw de ColombieBritannique en septembre 2003 à l'initiative d'Aube Elléouët-Breton et de sa fille Oona. À partir de l'analyse critique de l'histoire singulière de la coiffure, l'auteur met au jour les transformations de statut et de signification de l'objet ainsi que les enjeux politiques, culturels et esthétiques dans lequel il a été pris au cours de son histoire. L'exemple de la coiffure, et plus largement celui de la Potlatch Collection, montre qu'un objet extrait de son contexte d'origine ne retrouve généralement pas son statut initial, même dans le cadre de sa restitution à la communauté qui l'a fait naître.
Marie Mauzé, Three destinies, one fate. Biography of a Kwakwa ka'wakw headdress. - A ceremonial headdress which was part of the collection of surrealist poet André Breton was repatriated to the Kwakwaka'wakw of British Colombia in September 2003 following the initiative of Aube ElléouëtBreton and of her daughter Oona. Beginning with a critical analysis of the singular history of this headdress, the author brings to light the transformations of the object's status and meaning, as well as the political, cultural and aesthetic stakes which it has raised during the course of its existence. The example of the headdress and, more broadly, that of the Potlatch Collection, shows that once an object has been removed from its original context it does not generally retrieve its initial status even when it is repatriated to the community in which it originated. 